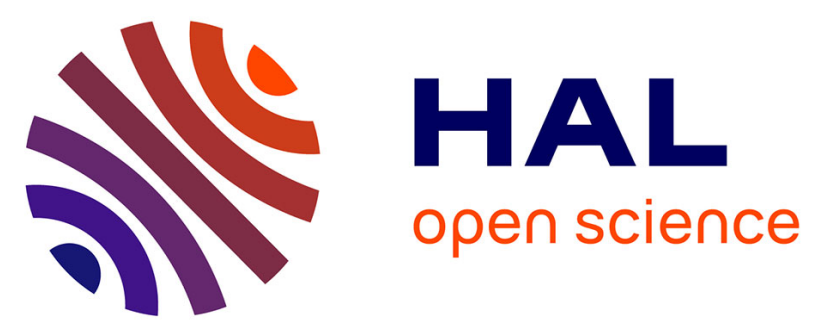

\title{
Farnesoid X Receptor Activation in Brain Alters Brown Adipose Tissue Function via the Sympathetic System
} Benjamin Deckmyn, Dorothée Domenger, Chloé Blondel, Sarah Ducastel, Emilie Nicolas, Emilie Dorchies, Emilie Caron, Julie Charton, Emmanuelle Vallez, Benoit Deprez, et al.

\section{To cite this version:}

Benjamin Deckmyn, Dorothée Domenger, Chloé Blondel, Sarah Ducastel, Emilie Nicolas, et al.. Farnesoid X Receptor Activation in Brain Alters Brown Adipose Tissue Function via the Sympathetic System. Frontiers in Molecular Neuroscience, 2022, 14, pp.808603. 10.3389/fnmol.2021.808603 . inserm- 03542610

\section{HAL Id: inserm-03542610 https://www.hal.inserm.fr/inserm-03542610}

Submitted on 25 Jan 2022

HAL is a multi-disciplinary open access archive for the deposit and dissemination of scientific research documents, whether they are published or not. The documents may come from teaching and research institutions in France or abroad, or from public or private research centers.
L'archive ouverte pluridisciplinaire HAL, est destinée au dépôt et à la diffusion de documents scientifiques de niveau recherche, publiés ou non, émanant des établissements d'enseignement et de recherche français ou étrangers, des laboratoires publics ou privés. 


\section{OPEN ACCESS}

Edited by:

Tiago F. Outeiro,

University Medical Center Göettingen,

Germany

Reviewed by:

Emmanuel Planel,

Laval University, Canada

Didier Vieau,

Université de Lille, France

Karelle Leroy,

Université Libre de Bruxelles, Belgium

*Correspondence:

Bart Staels

bart.staels@pasteur-lille.fr

Kadiombo Bantubungi kadiombo.bantubungi@inserm.fr

${ }^{\dagger}$ These authors have contributed equally to this work

Specialty section:

This article was submitted to

Brain Disease Mechanisms,

a section of the journal

Frontiers in Molecular Neuroscience

Received: 03 November 2021 Accepted: 07 December 2021

Published: 04 January 2022

Citation:

Deckmyn B, Domenger $D$, Blondel C, Ducastel S, Nicolas E, Dorchies E, Caron E, Charton J, Vallez E, Deprez B, Annicotte J-S, Lestavel S, Tailleux A, Magnan C, Staels B and Bantubungi K (2022)

Farnesoid X Receptor Activation in Brain Alters Brown Adipose Tissue

Function via the Sympathetic System.

Front. Mol. Neurosci. 14:808603.

doi: 10.3389/fnmol.2021.808603

\section{Farnesoid X Receptor Activation in Brain Alters Brown Adipose Tissue Function via the Sympathetic System}

Benjamin Deckmyn 1,2,3,4,5, Dorothée Domenger ${ }^{1,2,3,4}$, Chloé Blondel1,2,3,4, Sarah Ducastel 1,2,3,4, Emilie Nicolas ${ }^{1,2,3,4}$, Emilie Dorchies ${ }^{1,2,3,4}$, Emilie Caron ${ }^{6}$, Julie Charton ${ }^{4,7,8}$, Emmanuelle Vallez 1,2,3,4, Benoit Deprez ${ }^{4,7,8}$, Jean-Sébastien Annicotte ${ }^{9}$, Sophie Lestavel ${ }^{1,2,3,4}$, Anne Tailleux ${ }^{1,2,3,4}$, Christophe Magnan ${ }^{10,11}$, Bart Staels B, $^{1,3,4 * t}$ and Kadiombo Bantubungi ${ }^{1,2,3,4 * \dagger}$

${ }^{1}$ EGID, U1011, University of Lille, Lille, France, ${ }^{2}$ Inserm, U1011, Lille, France, ${ }^{3} \mathrm{CHU}$ Lille, Lille, France, ${ }^{4}$ Institut Pasteur de Lille, Lille, France, ${ }^{5}$ Laboratory of Lille Catholic Hospitals, Medical Biology Department, Lille Catholic University, Lille, France, ${ }^{6}$ Inserm UMR-S 1172, Lille, France, ${ }^{7}$ Inserm U1177, Lille, France, ${ }^{8}$ Drugs and Molecules for Living Systems, U1177, University of Lille, Lille, France, ${ }^{9}$ CNRS UMR 8199, Lille, France, ${ }^{10}$ CNRS UMR 8251, Paris, France, ${ }^{11}$ University Paris Diderot, Paris, France

The nuclear bile acid $(B A)$ receptor farnesoid $X$ receptor $(F X R)$ is a major regulator of metabolic/energy homeostasis in peripheral organs. Indeed, enterohepatic-expressed FXR controls metabolic processes (BA, glucose and lipid metabolism, fat mass, body weight). The central nervous system (CNS) regulates energy homeostasis in close interaction with peripheral organs. While FXR has been reported to be expressed in the brain, its function has not been studied so far. We studied the role of FXR in brain control of energy homeostasis by treating wild-type and FXR-deficient mice by intracerebroventricular (ICV) injection with the reference FXR agonist GW4064. Here we show that pharmacological activation of brain FXR modifies energy homeostasis by affecting brown adipose tissue (BAT) function. Brain FXR activation decreases the rate-limiting enzyme in catecholamine synthesis, tyrosine hydroxylase $(\mathrm{TH})$, and consequently the sympathetic tone. FXR activation acts by inhibiting hypothalamic PKACREB induction of TH expression. These findings identify a function of brain FXR in the control of energy homeostasis and shed new light on the complex control of energy homeostasis by BA through FXR.

\section{Keywords: FXR, brain, hypothalamus, energy homeostasis, brown adipose tissue}

\section{INTRODUCTION}

Proper energy homeostasis is crucial to maintain health and avoid the development of metabolic disorders such as obesity, dyslipidemia and type 2 diabetes. The essential role of the CNS in the regulation of energy homeostasis is now well documented (Bantubungi et al., 2012; Kim et al., 2018). The CNS closely interacts with peripheral organs to gather information on its energy state and provides, in turn, signals to adapt biological responses. Among the different brain regions controlling energy homeostasis, the hypothalamus is the major center of convergence and integration of nutrient/hormonal signals and environmental cues, particularly through two types of neurons, pro-opio-melanocortin (POMC)-producing neurons and agouti-related protein 
(AgRP)-producing neurons of the arcuate nucleus of hypothalamus (ARH). Moreover, the CNS communicates with peripheral organs, such as the liver, intestine and adipose tissue through the peripheral nervous system.

The Farnesoid X Receptor (FXR) belongs to the nuclear receptor superfamily of ligand-regulated transcription factors (Lefebvre et al., 2009). FXR is highly expressed in the liver and intestine, where it regulates the expression of target genes to control BA, glucose and lipid metabolisms (Lefebvre et al., 2009; Chávez-Talavera et al., 2017). Although expressed at much lower levels, a role for FXR has also been suggested in peripheral organs such as pancreas and adipose tissue (Cariou et al., 2007; Abdelkarim et al., 2010; Popescu et al., 2010; Seyer et al., 2013). FXR has also been involved in the control of energy homeostasis as exemplified by the reduction of body weight and adipose tissue mass in FXR-KO mice. In accordance, FXR deficiency was shown to protect from excessive weight gain in genetic and dietinduced (DIO) obesity models (Prawitt et al., 2011; Zhang et al., 2012), while FXR activation, by peripheral administration of GW4064, potentiated body weight gain and glucose intolerance in DIO mice (Watanabe et al., 2011). All studies investigating the metabolic control by FXR have so far exclusively focused on its action in peripheral organs (Lefebvre et al., 2009; Chávez-Talavera et al., 2017). While FXR reportedly is expressed in the brain (Gofflot et al., 2007; Huang et al., 2016), its function remains ill-defined. So far, studies evaluating BA-FXR signaling in the CNS mainly focused on its potential role in neurodegenerative conditions, such as Alzheimer's disease (Lo et al., 2013; Bell et al., 2018; McMillin et al., 2018), Parkinson's disease (Castro-Caldas et al., 2012; Abdelkader et al., 2016; Moreira et al., 2017; Rosa et al., 2018), Huntington's disease (Keene et al., 2001, 2002) as well as amyotrophic lateral sclerosis (Vaz et al., 2015; Elia et al., 2016). In these models of neurodegeneration, BA-FXR activation appears rather protective by acting on its pathophysiological mechanisms. Huang et al. showed alterations in depressive-like and anxiety-related behaviors in FXR-deficient mice, linked to an alteration of neurotransmitter homeostasis in different brain regions. The authors assumed that these effects are potentially mediated by changes in plasma and brain BA pools (Huang et al., 2015). Although the brain is well-recognized as an important regulator of peripheral homeostasis, whether brain FXR plays a role in the regulation of metabolism in peripheral organs remained unexplored. Given the importance of the CNS in the control of energy homeostasis and the role of FXR as a metabolic/energy sensor in peripheral organs, we investigated the role of brain FXR in the control of energy homeostasis. Here, we identify an unexpected novel function of brain FXR in its ability to control BAT function via a mechanism involving modification of hypothalamic PKA-CREB signaling and, subsequently, the sympathetic tone.

\section{MATERIALS AND METHODS}

\section{Animals}

Animal experiments were approved by the Institutional Committee for animal use and care. The ethical committee of the University of Lille approved all protocols (APAFIS\#1123720170911185145v2, APAFIS\#13331-2017091915214567v17). Male wild-type mice (C57BL/6J), male FXR-deficient mice [FXR-KO, provided by Sinal et al. (2000)] and their littermates (FXR-WT), on the C57BL/6J genetic background (Charles River), 16-19 weeks old, were housed under a $12 \mathrm{~h} / 12 \mathrm{~h}$ light/darkness cycle in temperature $\left(21.5^{\circ} \mathrm{C}\right)$ and humidity controlled rooms, in a specific pathogen-free environment. Standard diet (A04, Safe) and water were available ad libitum except during the cold-exposure experiment. Depending on the protocols, mice were either placed in metabolic cages (TSE systems, Hamburg, Germany) or standard cages and scarified in the fed status. All experiments were performed with minimum of 5 animals per group. The precise number of animals per group is mentioned in figure legends.

\section{Intracerebroventricular Cannulations}

Mice were randomized based on body weight, anesthetized using a mixture of ketamine $(75 \mathrm{mg} / \mathrm{kg}) / x y l a z i n e ~(10 \mathrm{mg} / \mathrm{kg})$, and stereotactically equipped with a cannula targeting the lateral ventricle of the brain (AP: $+0.24 \mathrm{~mm}, \mathrm{ML}:+1 \mathrm{~mm})$. The cannula was secured on the skull with dental ciment.

\section{Farnesoid X Receptor Agonist Treatments}

For intracerebroventricular (ICV) treatment, the synthetic FXR specific agonists GW4064 (3-(2,6-Dichlorophenyl)-4-(3'carboxy-2-chlorostilben-4-yl)-oxymethyl-5-isopropylisoxazole, Tocris, 2473/50, purity $>$ 97\%) and tropifexor (LJN452, Clinisciences) were dissolved in $100 \%$ DMSO and injected in a volume of $1 \mu \mathrm{l}, 0.25 \mu \mathrm{l} / \mathrm{min}$. In the chronic injection experiment, one ICV injection was administered every day (at the end of the day just before light turn off) for 6 days (GW4064 at the dose of $0.9 \mathrm{mM}$ or tropifexor at the dose of $0.2 \mathrm{mM}$ (doses selected after dose-response experiments in metabolic cages) or vehicle $(100 \%$ DMSO) and mice were sacrificed the day after the last injection. Peripheral blood concentration of GW4064 was determined by high-performance liquid chromatography combined with mass spectrometry (LC-MS/MS Acquity I-Class - Xevo TQD Waters).

\section{Mouse Monitoring in Metabolic Cages}

Wild-type mice were individually placed in metabolic cages (TSE systems, Hamburg, Germany). Energy parameters were recorded throughout the experiment: food intake (in grams, by minute and cumulative over $48 \mathrm{~h}$ ), locomotor activity (number of cage crossings, number of straightenings), $\mathrm{VO}_{2}$ and $\mathrm{CO}_{2}$ consumption (in $\mathrm{ml} / \mathrm{h} / \mathrm{kg}$ lean mass) was measured and energy expenditure calculated (Weir formula: $\mathrm{EE}=\left(3.94 \times \mathrm{VO}_{2}+1.106 \times \mathrm{VCO}_{2}\right) / 1000$ in $\mathrm{kcal} / \mathrm{h} / \mathrm{kg}$ lean mass) and the Respiratory Exchange Ratio (RER).

\section{Sympathetic Nerve Activity Recording}

Mice were anesthetized using a mixture of ketamine (75 $\mathrm{mg} / \mathrm{kg}) /$ xylazine $(10 \mathrm{mg} / \mathrm{kg})$, and the carotid artery was exposed. The sympathetic nerve filament was dissected free of underlying tissues on a distance of $1 \mathrm{~cm}$ until the superior 
cervical ganglion. The nerve was covered with paraffin oil and placed on a pair of recording silver electrodes ( $0.6 \mathrm{~mm}$ diameter) connected to a high-impedance probe, action potentials were saved after initial amplification through a low-noise amplifier (BIO amplifier, ADInstrument, Paris, France). Unipolar nerve activity was recorded continuously for $15 \mathrm{~min}$. Data were digitized with PowerLab/4sp digitizer (ADInstrument, Paris, France). Signals were amplified $105 \times$, filtered using low/highfrequency cut-offs of 100 and $1,000 \mathrm{~Hz}$, and monitored using the Chart 4 computer program (ADInstrument, Paris, France).

\section{Cold-Exposure Experiment}

C57BL/6J mice were individually housed at $4^{\circ} \mathrm{C}$ during $8 \mathrm{~h}$ without access to food and water during the cold-exposure period only. The rectal temperature was monitored using a rectal thermoprobe at the end of cold-exposure.

\section{RNA Extraction, cDNA Synthesis and Quantitative Real-Time PCR}

$\mathrm{PVH}, \mathrm{ARH}$, liver, BAT and eWAT were dissected and frozen in liquid $\mathrm{N}_{2}$. Total RNA was isolated using the RNeasy Lipid Tissue Mini Kit (Qiagen). Retrotranscription reactions were performed using the cDNA Reverse Transcription High Capacity Kit (Applied Biosystem). QPCR reactions were performed using Brilliant Sybr Green II QPCR Master Mix kit on the Stratagene MX3000P device (Agilent Technologies) or TaqMan Multiplex Master Mix (Applied Biosystem) on the Applied Biosystems 7500 Real Time PCR device. mRNA levels were normalized to a control gene (cyclophillin for Sybr green, $18 \mathrm{~S}$ for Taqman) whose expressions are not influenced by the experimental conditions. Supplementary Table 1 details the primer sequences used for real-time PCR.

\section{Western Blot Analysis}

Hypothalamus, BAT and liver were dissected and frozen in liquid $\mathrm{N}_{2}$. Tissues were homogenized in $1 \mathrm{ml}$ buffer (Trizmabase/sucrose) and sonicated. $20 \mu \mathrm{g}$ of protein in LDS sample buffer were loaded per lane, separated with NuPage 4-12\% Bis-Tris Protein Gels (Thermofischer, NP0335BOX) and transferred to nitrocellulose membrane iBlot 2 transfert stacks (Thermofischer, IB23001). Membranes were immunoblotted at $4^{\circ} \mathrm{C}$ overnight with antibodies against UCP1 (Abcam, AB10983, rabbit polyclonal, IgG; 1/500), Tyrosine Hydroxylase (Merck, MAB5280, mouse monoclonal, IgG; 1/500), FXR (Abcam Perseus, PP-A9033A-00, mouse monoclonal, IgG; 1/500), PKARII (BD Biosciences, 610626, mouse monoclonal, IgG; 1/250), PPKARII (BD Biosciences, 612550, mouse monoclonal, IgG; 1/250), HSP 90 $\alpha / \beta$ (H-114) (Santa cruz biotechnology, sc-7947, rabbit polyclonal, IgG; 1/1000) and $\beta$ Actine (Sigma, A5441, mouse monoclonal, IgG; 1/1000). All antibodies were diluted in Tris buffer saline (TBS) supplemented with Tween $0.01 \%$ and milk powder 5\%. The secondary antibodies used are goat anti mouse (Sigma A4416, IgG) or anti-rabbit (Sigma A0545, IgG), diluted in TBS supplemented with Tween 0.01\% and milk powder $5 \%$. The incubations are performed at room temperature $2 \mathrm{~h}$. Results are represented in the form of boxes for illustration purposes. All samples of an experiment were processed on the same western blot. When different gels were necessary, one or more common samples were run on each gel to allow subsequent normalization of the results.

\section{Immunohistochemistry Analysis}

Mice were anesthetized using a mixture of ketamine (75 mg/kg)/xylazine (10 mg/kg), followed by intracardiac perfusion using a solution of saline $(\mathrm{NaCl} 0.9 \%, 20 \mathrm{ml})$ and subsequently a solution of $4 \%$ PFA. BAT was fixed by immersion at $4^{\circ} \mathrm{C}$ in $4 \%$ PFA, dehydrated, cleared and embedded in paraffin. Paraffin sections (5 $\mu \mathrm{m}$ thick) were stained with hematoxylin and eosin. Brains were post-fixed for $16 \mathrm{~h}$ at $4^{\circ} \mathrm{C}$ in $4 \%$ PFA. Cryoprotection of brains was performed by successive $24 \mathrm{~h}$ baths of PBS-sucrose buffer $(10,20,30 \%)$ at $4^{\circ} \mathrm{C}$. Brains were included in Tissue Freezing Medium (Jung) before being frozen in isopentane cooled to $-55^{\circ} \mathrm{C}$ with liquid nitrogen. Blocks were stored at $-80^{\circ} \mathrm{C}$. Brains were cut at $18 \mu \mathrm{m}$ using a Leica cryostat (CM3050) and placed on Superfrost Plus slides (Thermofischer) for FXR (Abcam, AB28676, rabbit polyclonal, IgG; 1/100), $\alpha \mathrm{MSH}$ (Millipore AB5087, sheep polyclonal, IgG; 1/10000), HuD/C (Thermofischer, A-21271, mouse monoclonal, IgG; 1/500) staining. Free-floating slices of $40 \mu \mathrm{m}$ were prepared for $\mathrm{p}$-CREB immunohistochemistry. FXR-NPY co-staining was performed on mouse brain sections whose expression of the fluorescent GFP protein is under the control of the NPY promoter, not requiring double labeling. For FXR/ $\alpha \mathrm{MSH}$ and $\mathrm{FXR} / \mathrm{HuD} / \mathrm{C}$ co-staining, slides were incubated for $16 \mathrm{~h}$ at $4^{\circ} \mathrm{C}$ with FXR antibody diluted at $1 / 100$ (PBS $0.01 \mathrm{M}+1 \%$ blocking reagent), followed by an incubation for $2 \mathrm{~h}$ at room temperature with the secondary anti-rabbit antibody coupled to HRP (Thermofischer, B40922, goat polyclonal, IgG) diluted at 1/100 (PBS 0.01M $+1 \%$ blocking reagent). Next, the slides were incubated in biotinylated tyramide diluted at 1/100 (amplification buffer, Thermofischer, $\mathrm{B} 40922+\mathrm{H} 2 \mathrm{O} 2 \mathrm{0.0015 \%}$ ) for $5 \mathrm{~min}$ at room temperature. The second step was an incubation for $16 \mathrm{~h}$ at $4{ }^{\circ} \mathrm{C}$ in the presence of $\alpha \mathrm{MSH}$ antibody diluted at $1 / 10000$ (PBS $0.01 \mathrm{M}+0.1 \% \mathrm{X}-100$ newt $+1 \%$ donkey serum) or $\mathrm{HuD} / \mathrm{C}$ antibody diluted at $1 / 500$ (PBS $0.01 \mathrm{M}+0.1 \% \mathrm{X}-100$ newt $+1 \%$ goat serum). Then, slides were incubated for $2 \mathrm{~h}$ at room temperature with the secondary antibody coupled to a fluorochrome emitting at $555 \mathrm{~nm}$ (for MSH) (Molecular probe, A-21432, donkey polyclonal, IgG) or $568 \mathrm{~nm}$ (for $\mathrm{HuD} / \mathrm{C}$ ) (Invitrogen, A-31570, donkey polyclonal, IgG) diluted at $1 / 200$ (PBS $0.01 \mathrm{M}+0.1 \%$ newt X-100). Lastly, labeling was performed using a 1/1000 diluted Hoechst solution in $0.01 \mathrm{M}$ PBS (Invitrogen ${ }^{\circledR}$, Hoechst33258) and slides were mounted with lamellae (Dako ${ }^{\circledR}$ Fluorescent Mounting Medium).

For Free-floating immunohistochemistry, brain sections were placed in 12-wells culture plates and incubated for $16 \mathrm{~h}$ at $4^{\circ} \mathrm{C}$ with PCREB (Ser133) (87G3) (Cell Signaling, 9198S, rabbit polyclonal, IgG, goat polyclonal, IgG) antibody diluted at 1/1000 (PBS $0.01 \mathrm{M}+0.2 \%$ triton $\mathrm{X}-100+1 \%$ goat serum). Then, sections were incubated for $2 \mathrm{~h}$ at room temperature with the secondary antibody coupled to a fluorochrome emitting at $488 \mathrm{~nm}$ (Molecular probe, A11008) diluted at 1/1000 (PBS $0.01 \mathrm{M})$. Finally, labeling was performed using a 1/5000 diluted Hoechst solution and slides mounted on Superfrost Plus slides. 
The image acquisition was performed using a confocal microscope (LSM710, Zeiss). Quantification and analysis of signals were done using Imaris software. For quantitative analysis of p-CREB in ARH neurons, a total of 16951 neurons of control mice and 12704 neurons of treated mice were counted in $\mathrm{ARH}$ slices of independent control mice $(n=8)$ and GW4064-treated mice $(n=7)$, and the amount of pCREB was classified as either low, moderate or high based on the level of fluorescence intensity given by Imaris software.

\section{Hybridation in situ}

Fluorescent in situ hybridization was performed using RNAscope $^{\circledR} \quad$ Multiplex Fluorescent Reagent Kit 2.0 according to the manufacturer's instructions (Advanced Cell Diagnostics). Briefly, brain sections $(20 \mu \mathrm{m})$ were fixed in $4 \%$ paraformaldehyde for $1 \mathrm{~h}$ at $4^{\circ} \mathrm{C}$ and dehydrated through graded ethanol solutions (50, 70, and $100 \%$ ) for $5 \mathrm{~min}$ each. Sections were treated by hydrogen peroxyde reagent at room temperature for $10 \mathrm{~min}$ and then hybridized with probes at $40^{\circ} \mathrm{C}$ for $2 \mathrm{~h}$ in a humidified oven. The NR1H4 probe (FXR; Cat\# 484491), POMC probe (Cat\# 314081-C3) and the NPY probe (Cat\# 313321-C2) were used. After hybridization, brain sections were sequentially applied with a series of probe signal amplification steps (Opal 520, 570 and 690), rinsed with ACD wash buffer twice for 2 min between each step. Lastly, nuclear labeling was performed using a 1/1000 diluted Hoechst solution in 0.01M PBS (Invitrogen ${ }^{\circledR}$, Hoechst33258) and slides were mounted with lamellae (Dako ${ }^{\circledR}$ Fluorescent Mounting Medium).

\section{Statistical Analyses}

All values are reported as means \pm SEM. Data were analyzed using the unpaired Student's $t$, two-way ANOVA or $\mathrm{X}_{2}$ tests, using the Prism software (GraphPad, United States). Significance was set at $P<0.05$ for all experiments.

\section{Data Statement}

The data sets generated during and/or analyzed during the current study are available from the corresponding author upon reasonable request.

\section{RESULTS}

\section{Intracerebral Treatment With GW4064 Activates Brain Farnesoid X Receptor and Modifies Energy Homeostasis, Brown Adipose Tissue Function and the Sympathetic Tone}

To assess if brain FXR contributes to the regulation of energy homeostasis, we first fine-mapped the exact localization of FXR expression in the hypothalamus at mRNA and protein levels (Gofflot et al., 2007; Huang et al., 2016; Figures 1, 2). FXR expression was observed in neurons of the arcuate nucleus (ARH) (Figures 1B, 2B), expressed by both alphaMelanocyte-Stimulating Hormone and Neuropeptide Y ( $\alpha \mathrm{MSH}$ and NPY) neurons of the ARH (Figures 1B, 2C,D), which regulate energy homeostasis (Sánchez-Lasheras et al., 2010). To determine whether FXR plays a role in the brain control of energy homeostasis, we treated mice by ICV injection with the reference FXR agonist GW4064 (Maloney et al., 2000). Given the absence of precise data in the literature concerning the bioavailability and pharmacokinetics of GW4064 in the brain, we empirically determined the active dose by performing an effect/dose experiment in metabolic cages. Chow fed lean mice in metabolic cages received increasing doses of GW4064 by ICV administration and modification of metabolic parameters as well as FXR target genes expression were monitored. A dose of $0.9 \mathrm{mM}$; $522 \mathrm{ng} / \mu \mathrm{l} ; 1 \mu \mathrm{l}$ injected, of GW4064, was found to modify metabolic parameters and to increase the expression of established FXR target genes in the hypothalamus, indicative of a pharmacological activity and a cerebral activation of FXR. Indeed, mRNA levels of the FXR target genes Small Heterodimer Partner and Bile Salt Export Pump (Shp and Bsep) were induced in the ARH upon GW4064 treatment (Figures 3A,B), but not in the paraventricular nucleus of the hypothalamus (PVH) (Figures 4A,B), indicating specific activation of FXR in the ARH. No differences in respiratory exchange rate (RER), ambulatory activity, $\mathrm{Z}$ rearing (Figures $\mathbf{4 E - G}$ ) nor food intake (Figure 3C) were observed between GW4064 versus vehicle treated mice. However, GW4064 treatment slightly affected $\mathrm{VO}_{2}, \mathrm{VCO}_{2}$ (Figures 4C,D) and weakly but significantly decreased energy expenditure (EE) in the first part of the dark phase (Figure 3D). Moreover, after 6 days of GW4064 treatment, food efficiency was significantly enhanced along with an increased body weight gain, suggesting a positive energy balance (Figures 3E,F).

Because energy homeostasis is a dynamic balance between food intake and energy expenditure (Hall et al., 2012) (including physical activity, basal metabolism and thermogenesis), we next assessed whether brain FXR activation modified basal metabolism or thermogenesis. mRNA levels of genes involved in several pathways related to basal metabolism and thermogenesis were then measured in peripheral organs: liver, epididymal adipose tissue (eWAT) and BAT (Cannon, 2004). ICV treatment with GW4064 did not modify the expression of glucose nor lipid metabolism genes in liver nor in eWAT. Surprisingly, a decrease of Uncoupling Protein 1 (Ucp1) gene and protein expression (Figures 3G-I), a marker of BAT activity (Zhang and Bi, 2015), was found, suggesting that BAT function may be impaired upon CNS FXR activation. Moreover, a decrease of Vascular endothelial growth factor ( Vegf) gene expression (Supplementary Figure 1), a marker of vascularization and an enlargement of brown adipocytes (Figures 3J-L) were found upon GW4064 treatment, suggesting a remodeling of BAT (Shimizu et al., 2014). These effects of CNS FXR activation on BAT functions were not due to systemic leakage of the compound or the activation of peripheral FXR, since GW4064 could not be detected in plasma after ICV GW4064 injection using a highly sensitive analytical system $[\mathrm{C}<10 \mathrm{nM}(\mathrm{LOQ})]$ nor were any of the classical FXR target genes induced in livers of these mice.

To understand the action mechanism of CNS FXR on energy expenditure and BAT function, the brain-BAT axis was next investigated by assessing the impact of FXR on the adrenergic tone exercised by the peripheral nervous system. Indeed, 
A

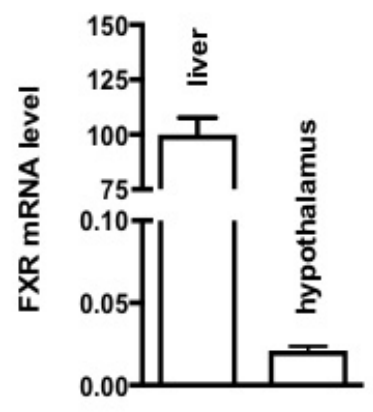

B
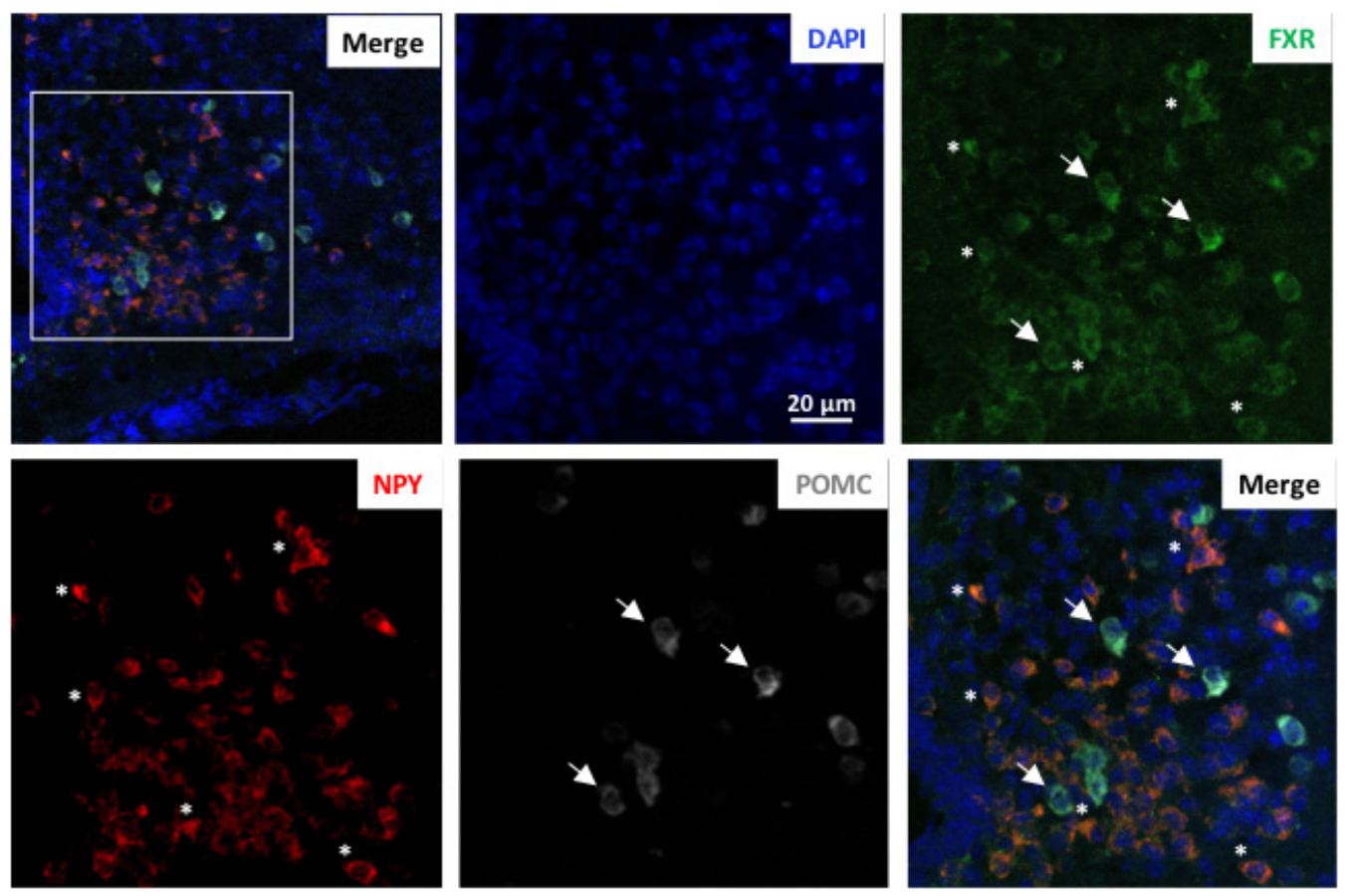

FIGURE 1 | FXR mRNA is expressed in hypothalamus. (A) Fxr mRNA expression in liver and hypothalamus by qPCR. The values are normalized to cyclophilin. Results are expressed by comparing the expression of FXR in the liver, whose expression level has been arbitrarily set at 100\%. (B) Representative images of RNAscope staining of FXR with NPY and POMC in ARH. The nuclear staining (blue) was performed with Hoechst solution. 3V, third ventricle; ARH, arcuate nucleus of hypothalamus; ME, median eminence.

BAT contains peripheral sympathetic fibers expressing Tyrosine Hydroxylase (TH) (Razzoli et al., 2016), the rate-limiting enzyme in catecholamine synthesis. These $\mathrm{TH}$-positive fibers release noradrenaline which through $\beta-3$ adrenergic receptors induces intracellular signaling increasing UCP1 expression in BAT (Harms and Seale, 2013). Interestingly, in mice ICV treated for 6 days with GW4064, TH expression in afferent BAT adrenergic neurons significantly decreased (Figures 3M,N). Moreover, the activity of the sympathetic nervous system was significantly decreased in mice treated ICV with GW4064 as compared to vehicle controls (Figure 30). Thus, CNS FXR activation by GW4064 impairs the sympathetic tone in BAT.

To ascertain the engagement of brain FXR on energy homeostasis and BAT, additional experiments were performed using a different synthetic FXR agonist, tropifexor, which is currently undergoing phase 2 human clinical trials in NASH and PBC (Tully et al., 2017). Noteworthy, ICV treatment with tropifexor also resulted in a decrease of $\mathrm{EE}$ without impacting food intake (Figures 5A,B). Moreover, icv treatment with tropifexor also increased BAT lipid droplet size (Figures $5 \mathrm{C}-\mathrm{E}$ ), indicating that CNS FXR activation affects the BAT remodeling.

\section{Intracerebral GW4064 Treatment Lowers Brown Adipose Tissue Activity and Rectal Temperature}

To determine whether central FXR activation functionally impacts on BAT function, the effect of ICV GW4064 


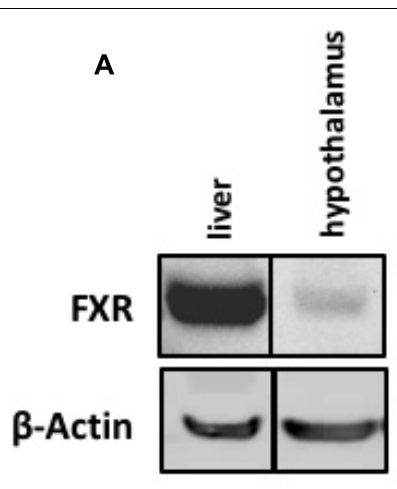

B
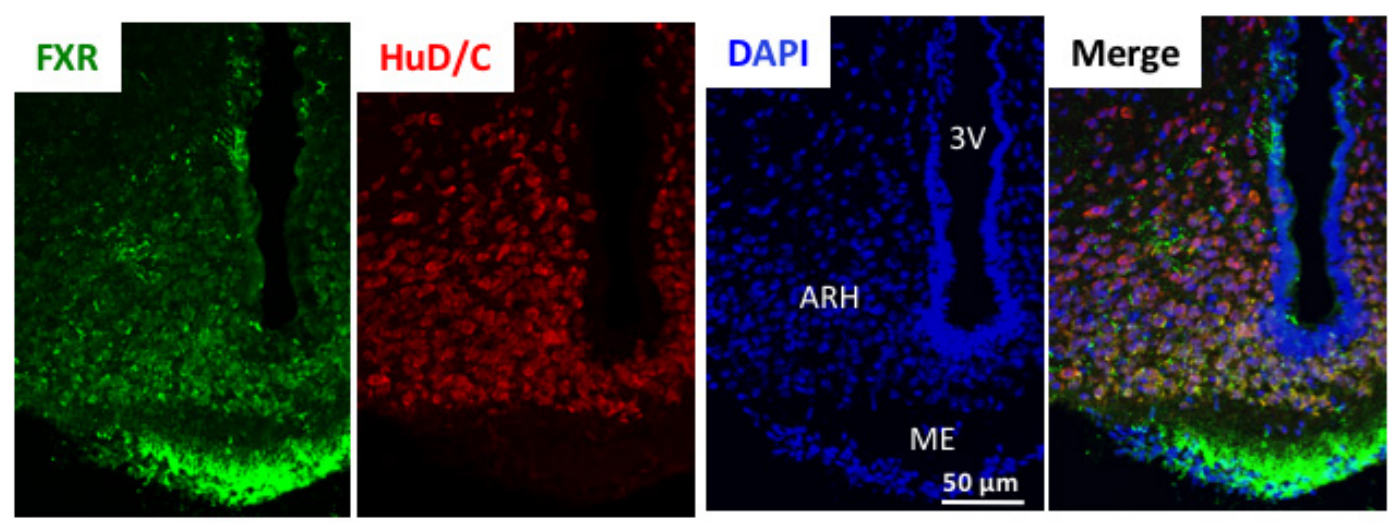

C

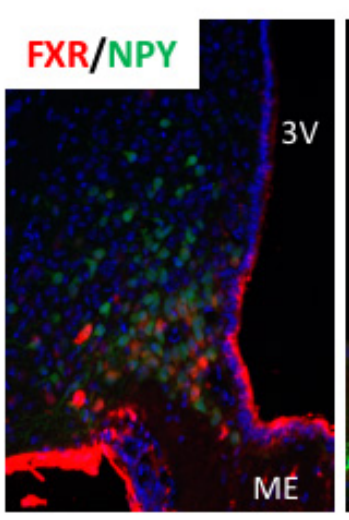

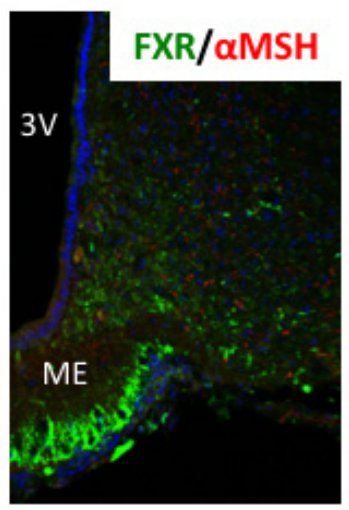

D

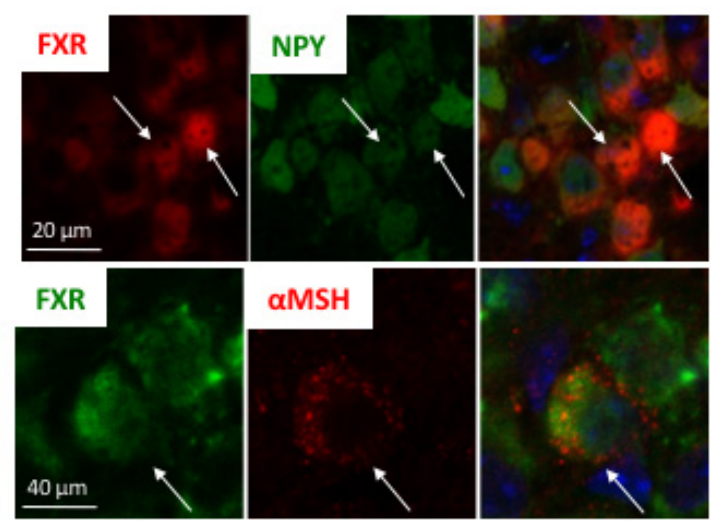

FIGURE 2 | FXR protein is expressed in hypothalamus. (A) FXR protein expression in liver and hypothalamus by Western blot. (B-D) Representative images of co-immunostaining of FXR with HuD/C (B) or NPY or aMSH (C) in ARH. (D) Higher magnification of panel (D). The nuclear staining (blue) was performed with Hoechst solution. $3 \mathrm{~V}$, third ventricle; $\mathrm{ARH}$, arcuate nucleus of hypothalamus; ME, median eminence.

pretreatment on adaptive thermogenesis was studied by subjecting mice to $8 \mathrm{~h}$ cold exposure. As expected, cold exposure induced Ucp 1 and Th mRNA and protein levels in vehicle-treated mice (Figures 6A-E). Surprisingly, ICV GW4064 pretreatment severely blunted this induction (Figures 6A-E). Moreover, rectal temperature was significantly lower in mice pretreated ICV with GW4064 as compared to vehicle when placed at $4^{\circ} \mathrm{C}$ (Figure 6F). Cold exposure is a major stimulus leading to increased metabolic functions in BAT (Harms and Seale, 2013). As expected, mRNA levels of the $\alpha 1-, \alpha 2$ - and $\beta 3$-adrenergic receptors in BAT as well as Peroxisome Proliferator-Activated Receptor Gamma Coactivator 1-alpha and Iodothyronine Deiodinase 2 (Pgcla and Dio2) were increased in response to $8 \mathrm{~h}$ cold exposure in BAT (Supplementary Figures 2A-E). However, ICV treatment with GW4064 did not modulate the $8 \mathrm{~h}$ cold exposure-induced expression of these genes, suggesting a mechanism upstream 
A

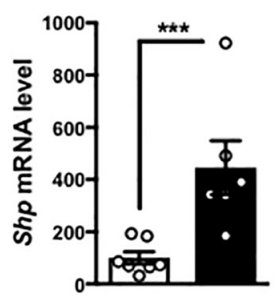

B

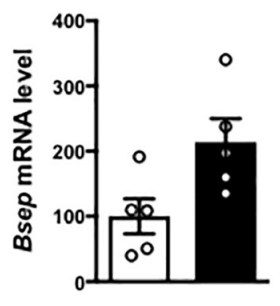

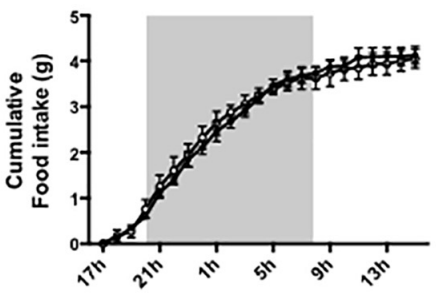

D

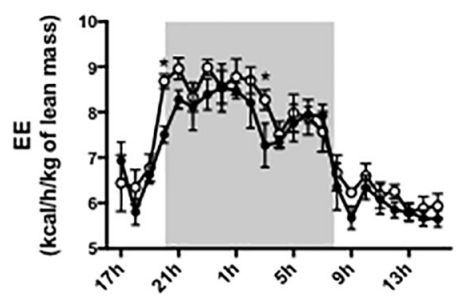

E

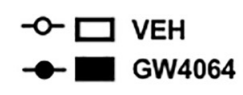

F

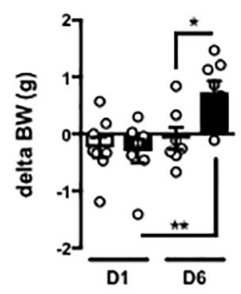

G

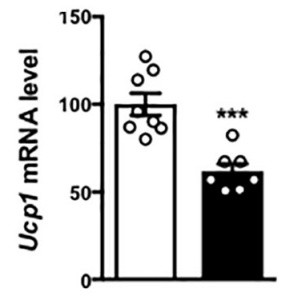

H

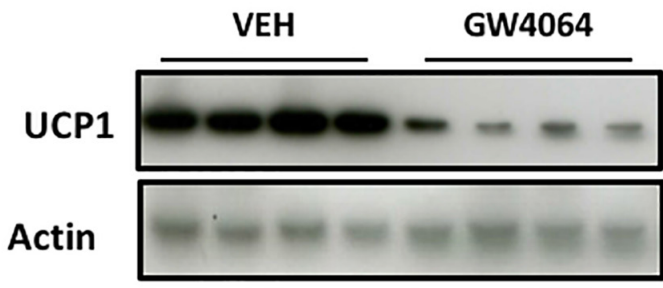

I

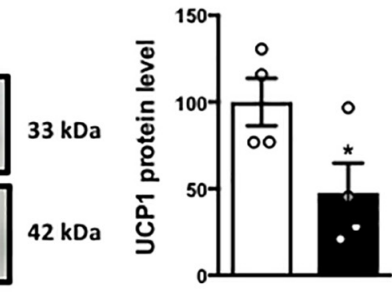

L

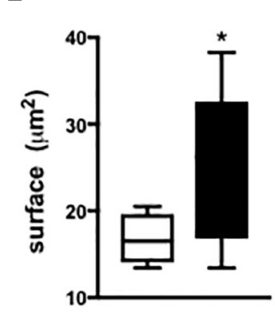

M
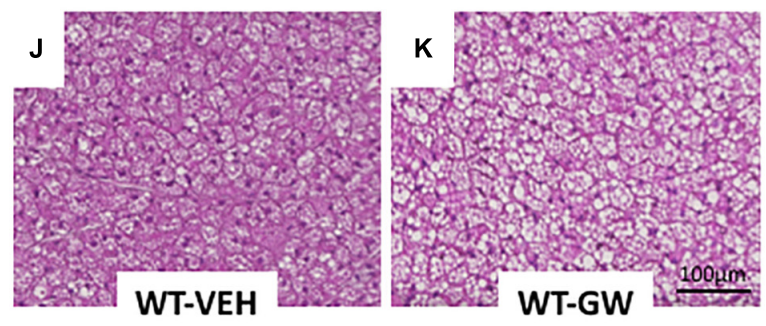

$\mathbf{N}$

0
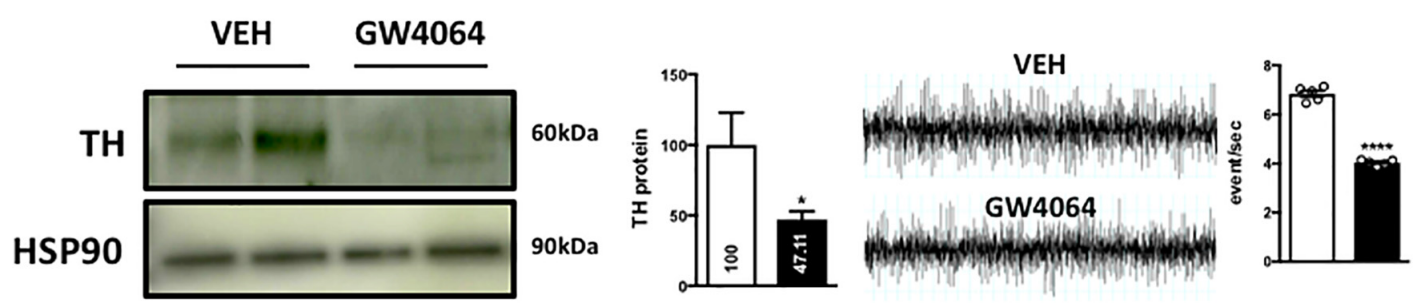

FIGURE 3 | Intracerebral treatment with GW4064 activates brain FXR and modifies energy homeostasis, BAT function and the sympathetic tone. (A,B) Shp and Bsep mRNA expression in ARH by q-PCR. The values are normalized to cyclophilin. (C) Cumulative food intake and (D) energy expenditure (EE) were measured in metabolic cages. (E) Food efficiency was calculated by ratio of body weight and food intake after one day and 6 days of treatment by GW4064. (F) Body weight gain was measured after one day and 6 days of treatment. (G) Ucp1 mRNA expression in BAT by q-PCR. The values are normalized to cyclophilin. (H) UCP1 protein expression in BAT. (I) The bar graph is the quantification of UCP1 western blots in panel (H). (J,K) Representative images of histological hematoxylin and eosin (H\&E) staining of BAT. (L) Quantification of lipid droplet surface. (M) TH protein expression in BAT. (N) The bar graph is the quantification of TH western blots in panel (M). (O) Representative recordings of sympathetic nerve activity from VEH and GW4064 treated mice and histogram of sympathetic nerve activity. Data are mean \pm SEM. ${ }^{*} P<0.05,{ }^{\star \star} P<0.01,{ }^{* \star *} P<0.001,{ }^{\star \star \star \star} P<0.0001$, Unpaired Student's $t$ test or Two-Way ANOVA followed by Tukey post hoc. Vehicle group is indicated as open circles/bars, GW4064 group as black circles/bars. 

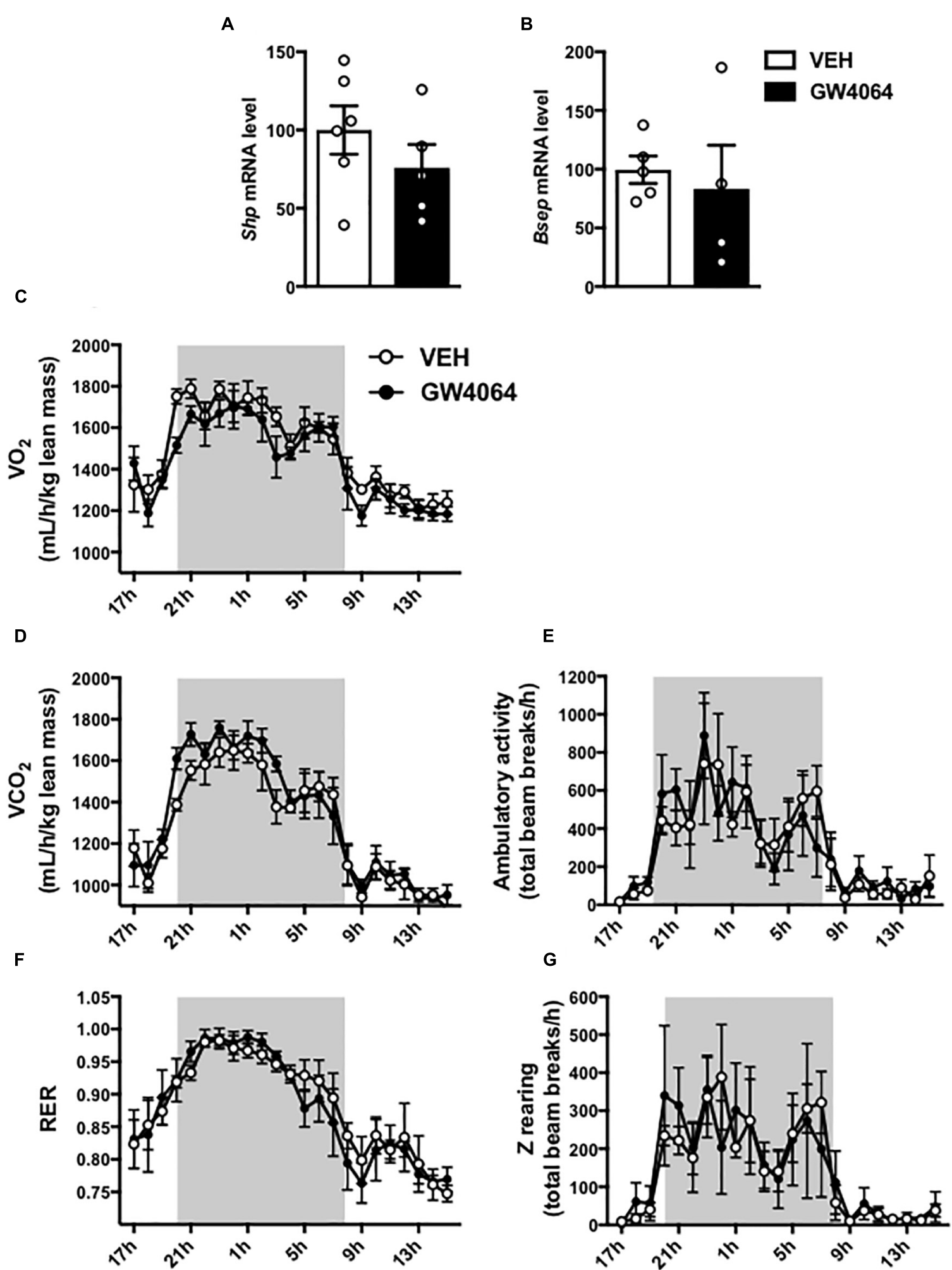

FIGURE 4 | Effect of 6-days cerebral treatment with GW4064 on FXR target gene expression in PVH and energy homeostasis. (A,B) Shp and Bsep mRNA expression in PVH by q-PCR. The values are normalized to cyclophilin. (C) $24 \mathrm{~h}$-oxygen consumption $\left(\mathrm{VO}_{2}\right)$. (D) $24 \mathrm{~h}$-carbon dioxide consumption.

(E) $24 \mathrm{~h}$-spontaneous locomotor activity (total beam breaks/hour). (F) $24 \mathrm{~h}$-respiratory exchange ratio (RER). (G) 24 h-spontaneous Z-rearing (total beam breaks/hour). Data are mean \pm SEM. Unpaired Student's $t$ test or Two-Way ANOVA followed by Tukey post hoc. Vehicle group is indicated as open circles/bars, GW4064 group as black circles/bars. 


\section{- $\square$ VEH \\ $\rightarrow$ TROPIFEXOR}

A

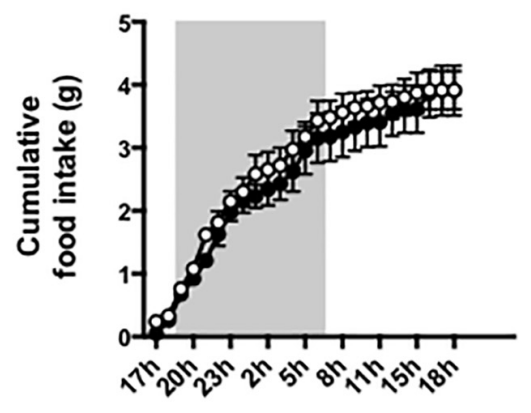

B

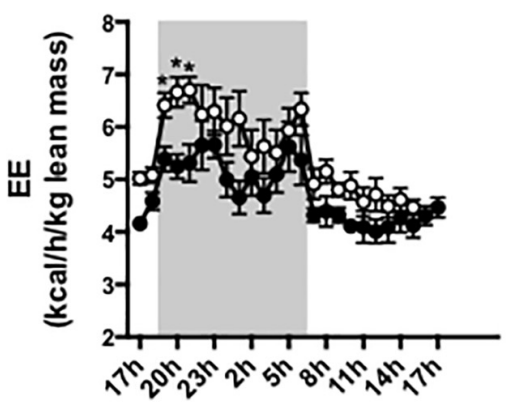

E
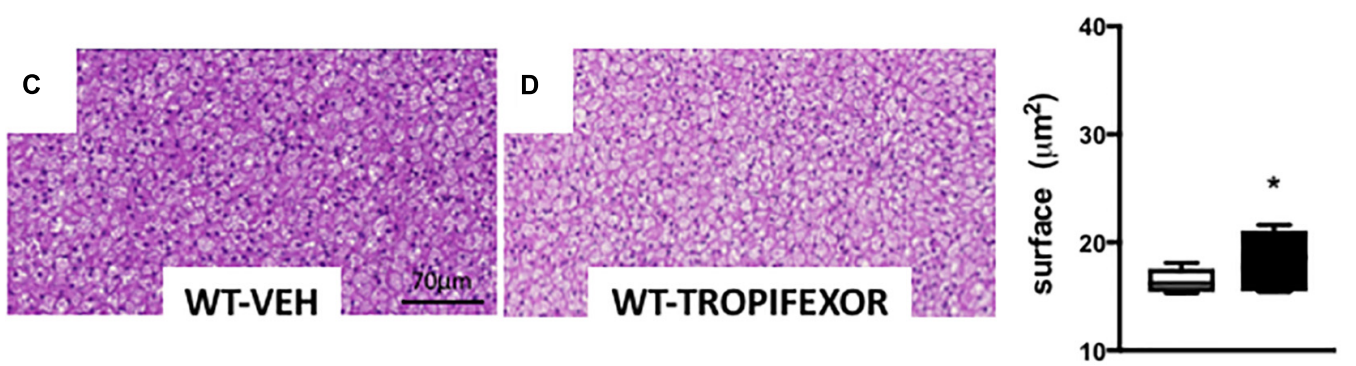

FIGURE 5 | Intracerebral treatment with tropifexor modifies energy homeostasis and BAT function. (A) Cumulative food intake and (B) energy expenditure (EE) were measured in metabolic cages. (C,D) Representative images of histological hematoxylin and eosin (H\&E) staining of BAT. (E) Quantification of lipid droplet surface. Data are mean \pm SEM. ${ }^{*} P<0.05$, Two-Way ANOVA followed by Tukey post hoc. Vehicle group is indicated as open circles/bars, GW4064 group as black circles/bars.

of adrenergic receptor signaling and excluding a mechanism dependent on thyroid signaling.

\section{Intracerebral GW4064 Treatment Alters Protein Kinase-c-AMP Response Element-Binding Protein Signaling and Tyrosine Hydroxylase Expression in the Hypothalamus}

To determine how cerebral FXR activation regulates the sympathetic tone in BAT, TH expression was measured in the hypothalamus. TH neurons are located in the PVH of the hypothalamus and project to the brainstem and spinal cord autonomic regulatory centers to integrate sympathetic outflow, for example to BAT (Swanson and Sawchenko, 1983). Interestingly, ICV GW4064 treatment decreased Th mRNA and TH protein expression in the hypothalamus (Figures 7AC). $\mathrm{TH}$ expression in the hypothalamus is under control of the transcription factor c-AMP Response Element-Binding protein (CREB) upon its phosphorylation by c-AMP-dependent Protein Kinase (PKA) (Piech-Dumas and Tank, 1999), through interaction with PKARII in the hypothalamus (Yang and McKnight, 2015). Interestingly, PKARII protein phosphorylation was lower in the hypothalamus upon ICV GW4064 treatment (Figures 7D,E), which was associated with a decrease in pCREB immunostaining in the ARH (Figures $7 \mathbf{F}-\mathbf{H}$ ). Moreover, ICV 

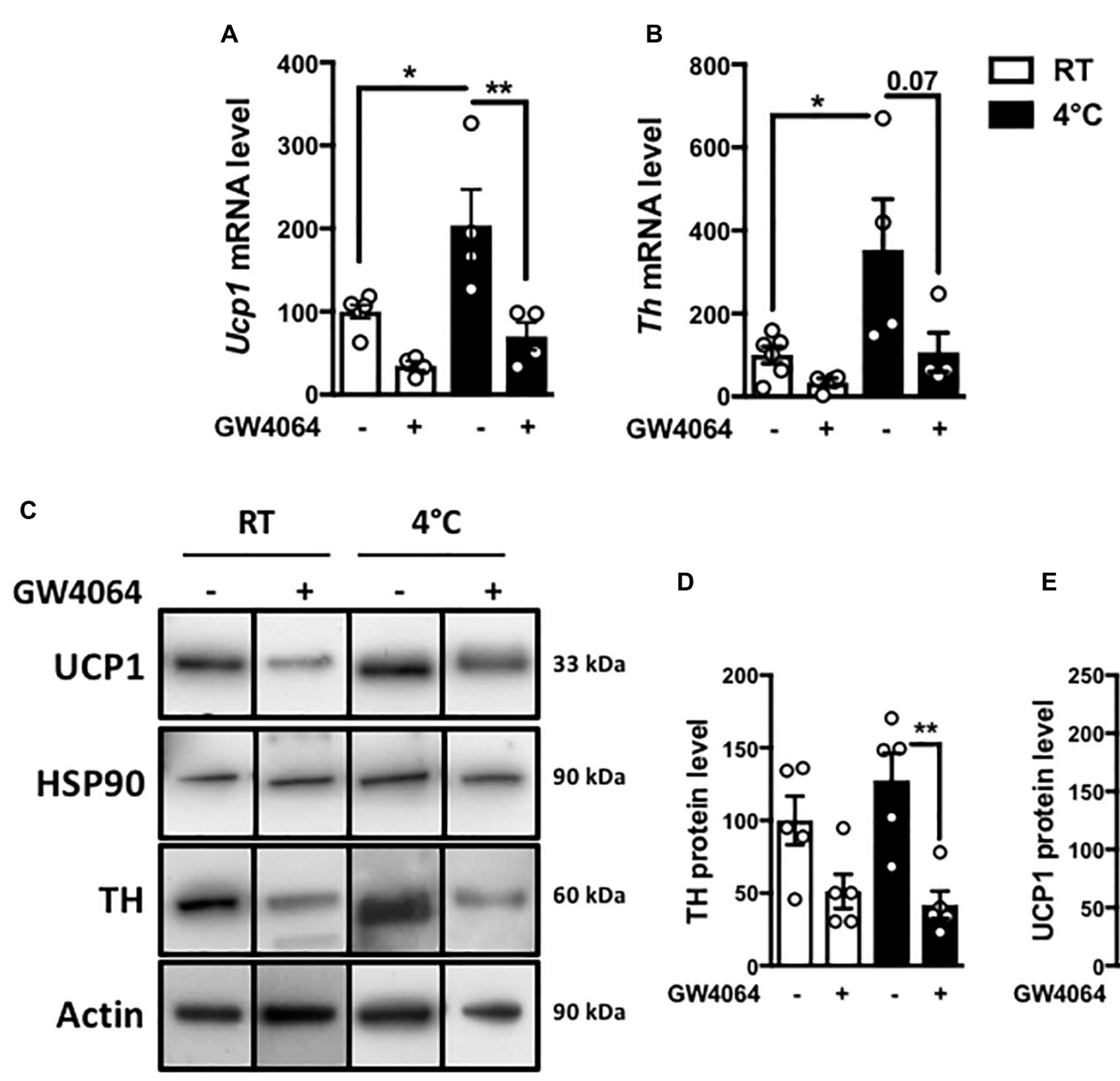

D

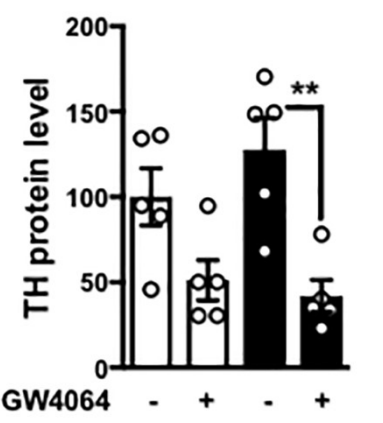

E

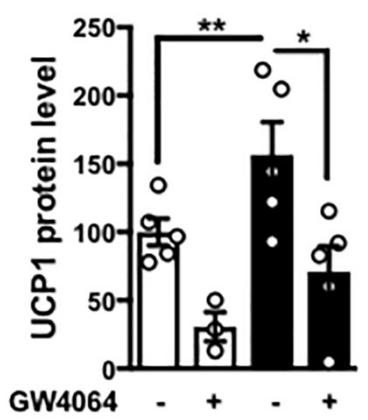

F

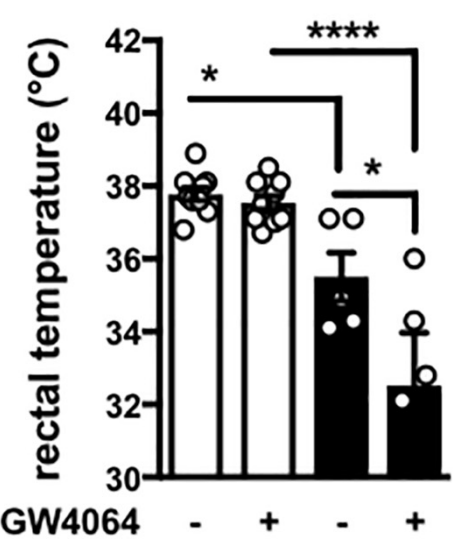

FIGURE 6 | Intracerebral treatment with GW4064 functionally impacts the BAT and rectal temperature. (A) Ucp1 mRNA expression in BAT of mice placed at room temperature $\left(23^{\circ} \mathrm{C}\right)$ or $4^{\circ} \mathrm{C}$. (B) Th mRNA expression in BAT of mice placed at room temperature $\left(23^{\circ} \mathrm{C}\right)$ or $4^{\circ} \mathrm{C}$. The values are normalized to cyclophilin or $18 \mathrm{~S}$. (C) UCP1 and TH protein expression in BAT of mice placed at room temperature $\left(23^{\circ} \mathrm{C}\right)$ or $4^{\circ} \mathrm{C}$. Results are represented in the form of boxes for illustration purposes. For an experiment, all samples are processed in the same western blot. If different gels were used if the number of wells was insufficient, we took the precaution of introducing one or more common samples within each gel to standardize the results. (D) The bar graphs are the quantification of UCP1 western blots in panel (C). (E) The bar graph is the quantification of TH western blots in panel (C). (F) Rectal temperature was measured at the end of $23^{\circ} \mathrm{C}$ - or 4 h-cold exposure. Data are mean \pm SEM. ${ }^{\star} P<0.05,{ }^{\star \star} P<0.01,{ }^{\star \star \star \star} P<0.0001$, Two-Way ANOVA followed by Tukey post hoc. $23^{\circ} \mathrm{C}$ group is indicated as open bars, $4^{\circ} \mathrm{C}$ group as black bars. 


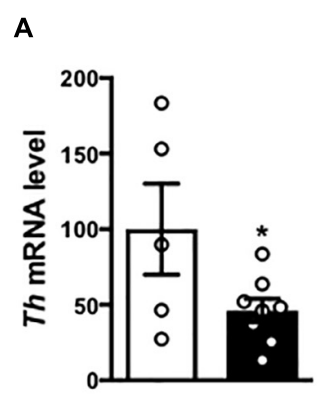

B

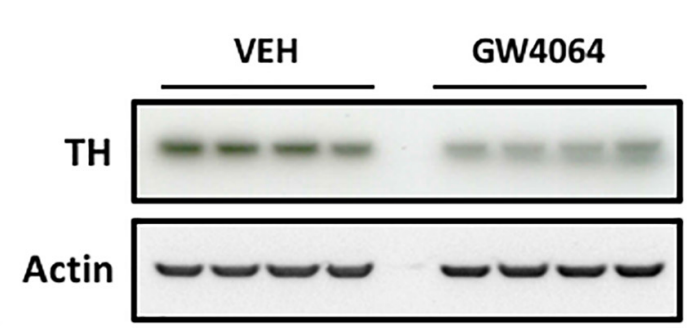

C

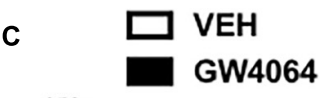

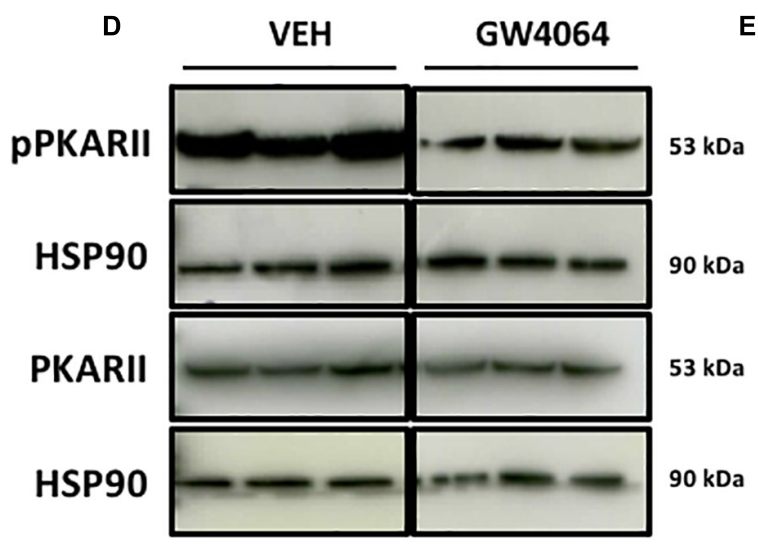

E

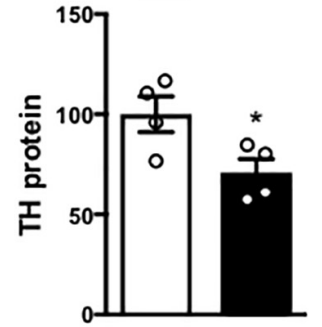

$60 \mathrm{kDa}$

$42 \mathrm{kDa}$

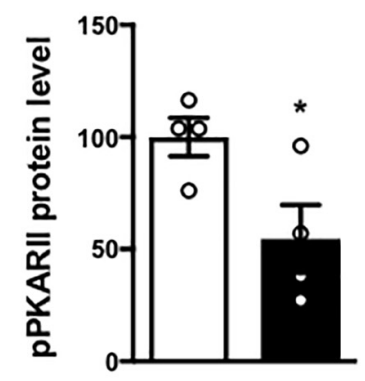

$\mathbf{F}$

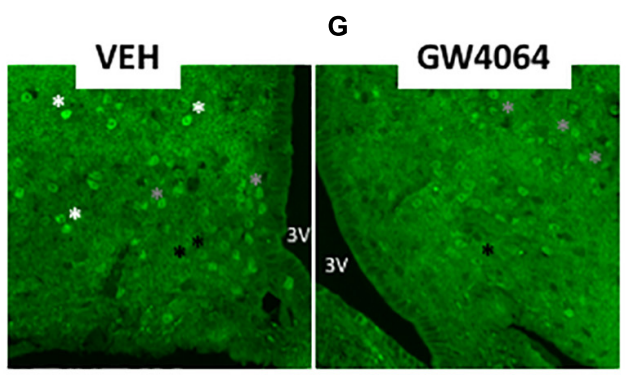

H

\begin{tabular}{|c|c|c|c|}
\hline & low & moderate & high \\
\hline VEH & 18116 & 14157 & 2414 \\
\hline GW4064 & 17792 & 8705 & $307 * * *$ \\
\hline
\end{tabular}
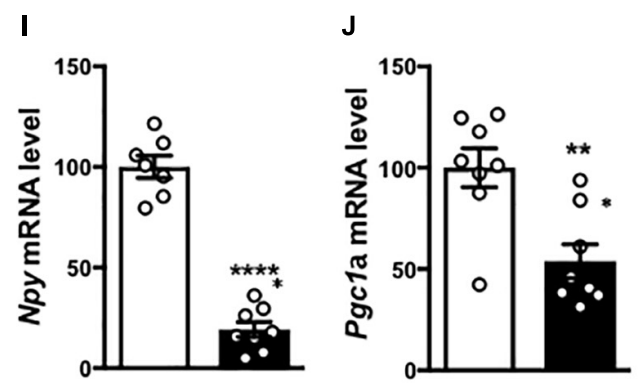

FIGURE 7 | Intracerebral treatment with GW4064 alters PKA-CREB signaling and TH expression in the hypothalamus. (A) Th mRNA expression in hypothalamus by q-PCR. The values are normalized to $18 \mathrm{~s}$. (B) TH protein expression in hypothalamus. (C) The bar graphs are the quantification of TH western blots in panel (B). (D) pPKARII and PKARII protein expression in hypothalamus. Results are represented in the form of boxes for illustration purposes. For an experiment, all samples are processed in the same western blot. If different gels were used if the number of wells was insufficient, we took the precaution of introducing one or more common samples within each gel to standardize the results. (E) The bar graphs are the quantification of pPKARIl western blots in panel (D). (F,G) Representative images of pCREB immunostaining in ARH of vehicle group (F) and GW4064 group (G). (H) Quantification of pCREB-positive cells with a low (black asterisk), moderate (gray asterisk), and high staining (white asterisk). (I,J) Npy and Pgc1a mRNA expression in ARH. The values are normalized to cyclophilin. Data are mean \pm SEM. ${ }^{\star} P<0.05,{ }^{\star \star} P<0.01,{ }^{\star \star \star} P<0.001,{ }^{\star \star \star \star *} P<0.0001$, Unpaired Student's $t$ test. For pCREB quantification, $X 2$ test was performed. Vehicle group is indicated as open bars, GW4064 group as black bars. 
A

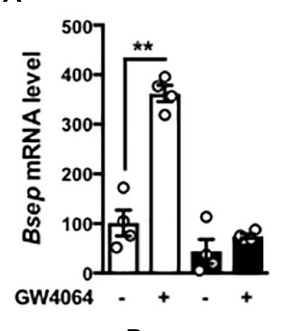

C

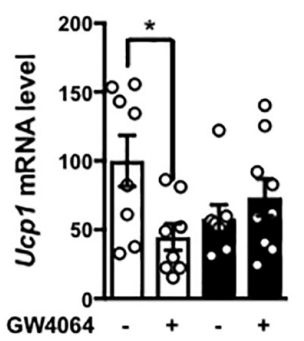

F

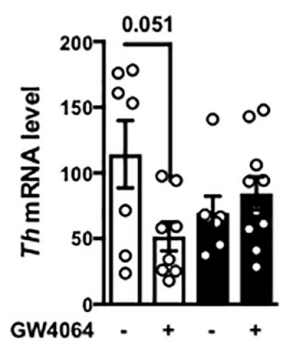

B

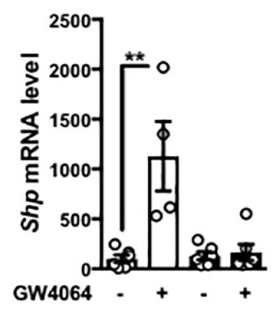

D

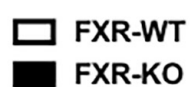

E

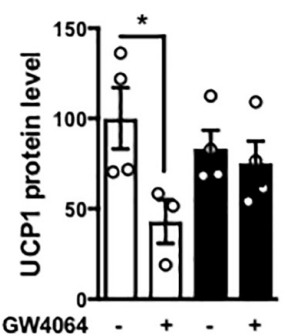

H

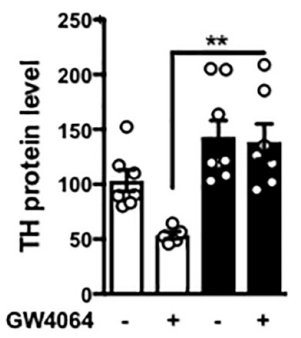

I

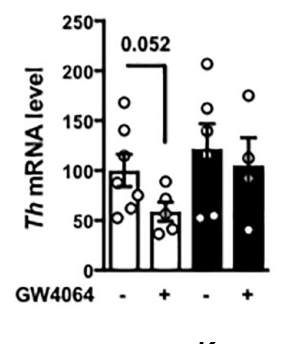

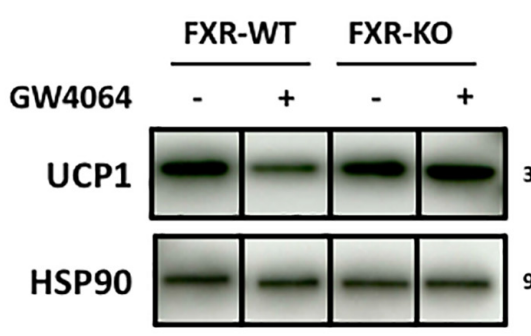

G

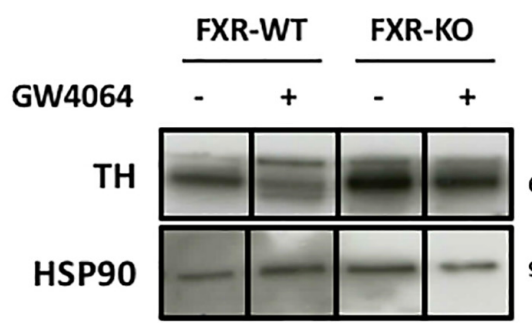

$60 \mathrm{kDa}$

$90 \mathrm{kDa}$

$33 \mathrm{kDa}$

$90 \mathrm{kDa}$

\section{J}

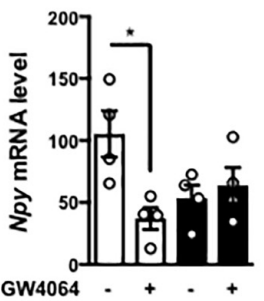

L

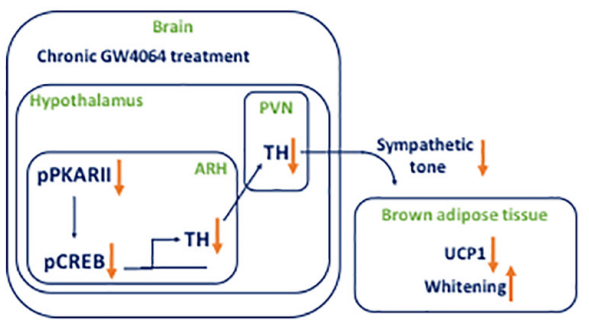

FIGURE 8 | FXR mediates the effects of intracerebral treatment with GW4064 on BAT. (A) Besp, (B) Shp and (I) Th mRNA expression in hypothalamus of FXR-WT and FXR-KO mice receiving vehicle solution or GW4064 by ICV injection. The values are normalized to cyclophillin or $18 \mathrm{~s}$. (C) Ucp1 mRNA expression in BAT of FXR-WT and FXR-KO mice receiving vehicle solution or GW4064 by ICV injection. The values are normalized to cyclophilin. (D) UCP1 protein expression in BAT. (E) The bar graphs are the quantification of UCP1 western blots in panel (D). (F) Th mRNA expression in BAT of FXR-WT and FXR-KO mice receiving vehicle solution or GW4064 by ICV injection. The values are normalized to $18 \mathrm{~s}$. (G) TH protein expression in BAT. Results are represented in the form of boxes for illustration purposes. For an experiment, all samples are processed in the same western blot. If different gels were used if the number of wells was insufficient, we took the precaution of introducing one or more common samples within each gel to standardize the results. $(\mathbf{H})$ The bar graphs are the quantification of TH western blots in panel (G). (J,K) Npy and Pgc1a mRNA expression in ARH. The values are normalized to cyclophilin. Data are mean \pm SEM. ${ }^{*} P<0.05$, ${ }^{* \star} P<0.01$, Two-Way ANOVA followed by Tukey post hoc. FXR-WT group is indicated as open bars, FXR-KO group as black bars. (L) Model: Hypothalamic activation of FXR decreases the activity of PKA-CREB in the hypothalamus, leading to a decrease of hypothalamic TH expression, which disrupts brain-BAT axis. 
GW4064 treatment decreased mRNA levels of the well-known CREB target genes Npy and Pgc1a (Herzig et al., 2001; Pandey, 2003) in the ARH (Figures 7/,JJ). These data demonstrate that pharmacological activation of hypothalamic FXR alters hypothalamic PKA-CREB signaling, hence modulating $\mathrm{TH}$ expression in the hypothalamus, and ultimately reducing the sympathetic tone on BAT, thus affecting energy homeostasis.

\section{Farnesoid X Receptor Mediates the Effects of Intracerebral GW4064 Administration on Brown Adipose Tissue}

To ensure the FXR dependence of the response to ICV GW4064, we next investigated the impact of ICV GW4064 in FXRdeficient mice and their littermate controls. The induction of FXR target genes (Bsep and $S h p$ ) in the ARH upon ICV GW4064 treatment in wild-type (FXR-WT) mice was not observed in FXR-deficient (FXR-KO) mice (Figures 8A,B). Moreover, the effect of ICV GW4064 treatment on Ucp1 (Figures 8C-E) and TH (Figures $\mathbf{8 F}-\mathbf{H}$ ) protein expression in BAT observed in FXR-WT mice was not observed in FXR-KO mice. Furthermore, the reduction of hypothalamic Th, Npy and Pgcla mRNA gene expression observed in FXR-WT mice upon ICV GW4064 treatment was also not observed in FXR-KO mice (Figures 8I-K). These data collectively identify a role of FXR in brain control of energy homeostasis through a brain-BAT axis involving hypothalamic FXR whose activation decreases energy expenditure (Figure 8L).

\section{DISCUSSION}

FXR is a key regulator of entero-hepatic metabolism. Besides its action on BA, lipid and glucose metabolism, FXR regulates insulin sensitivity and GLP1 secretion (Trabelsi et al., 2015; Chávez-Talavera et al., 2017). Moreover, whole body FXR deficiency induces a resistance to diet or genetic induced obesity (Prawitt et al., 2011; Zhang et al., 2012). Interestingly, we found that FXR is expressed in the hypothalamus, a brain area playing a crucial role in the regulation of energy homeostasis (Waterson and Horvath, 2015; Kim et al., 2018). To investigate whether brain FXR activation controls energy homeostasis, brain FXR was pharmacologically activated using the selective FXR agonist GW4064. Our results demonstrate that activation of brain FXR by ICV GW4064 administration leads to histological remodeling of BAT (i.e., increase of lipid droplet size) through hypothalamic regulation of the sympathetic tone. Importantly, these changes promoted by ICV FXR agonist treatment were absent in FXR$\mathrm{KO}$ mice indicating target-selectivity of the compound. In line with previous findings that GW4064 activates the expression of Shp, a known FXR target gene, in primary neuronal cultures (Huang et al., 2016), we found that ICV administrated GW4064 also induces its target genes in vivo (Figures 3A,B), in a FXRdependent manner (Figures 8A,B).

Our results showed altered BAT adrenergic activity (decreased $\mathrm{TH}$ protein expression), associated with a decrease of sympathetic tone activity, upon ICV GW4064 administration (Figure 30). The metabolic cage analysis did not allow assessment of possible consequences of the altered autonomic nervous system activity on major cardiovascular functions (heart rate, contractility, arterial pressure). Nevertheless, after 6 days of ICV GW4064 treatment, we observed slight decreases in $\mathrm{O}_{2}$ and increases in $\mathrm{CO}_{2}$ consumption at night. These observations are in line with a modified sympathetic tone, since disorders of sympathetic control or modulation of adrenergic receptors are known to impact $\mathrm{O}_{2}$ and $\mathrm{CO}_{2}$ consumption (Narkiewicz et al., 2006; Houssiere et al., 2007; Witte et al., 2008)(Ek and Åblad, 1971; Lang et al., 1989; Billinger et al., 2001; Witte, 2003).

The limited treatment duration ( 6 days) may explain the relatively mild, albeit significant metabolic changes observed in the metabolic cages. However, the effects on BAT are substantial as demonstrated by the pronounced changes in UCP1 protein expression, BAT histology and response upon cold exposure (Figures 3G-L, 6). Further studies are required to assess the impact of CNS FXR activation on energy metabolism in models of chronically altered energy homeostasis, such as in diet-induced obesity. Such studies are, however, difficult to perform given the invasive nature of the ICV delivery of GW4064.

Even though the ICV administered amounts of GW4064 are many-fold lower than the amounts typically given per os or $i p$, to exclude possible effects of peripheral leakage of GW4064 following ICV injection, plasma GW4064 concentrations were measured using a highly sensitive analytical system (high performance liquid chromatography coupled with mass spectrometry). This assay revealed undetectable GW4064 amounts in the plasma of mice treated 6d with ICV GW4064. Moreover, none of the classical FXR target genes were found to be induced in the liver arguing against a systemic leakage of the compound and/or a peripheral activation of FXR (Supplementary Figures 3A,B). Finally, whereas BAT has been shown to express the G-protein coupled membrane BA receptor TGR5, expression of FXR was not detectable by western blot analysis, confirming previous reports (Watanabe et al., 2006). Moreover, GW4064 has no activity on TGR5, ruling out such off-target effects (Maloney et al., 2000). All these data exclude the possibility that the observed alteration of BAT function is due to a peripheral activation of FXR induced by a leakage of GW4064 (from the brain to the periphery).

Peripheral administration of GW4064 has been shown to alter hepatic expression of genes involved in autophagy through mechanisms implicating a cross-talk with CREB (Seok et al., 2014). Our data indicate that activation of FXR in the brain acts through a similar mechanism: inhibition of the PKACREB pathway in the ARH leading to a decrease of CREB target gene expression. Although we have shown that central pharmacological activation of FXR alters PKA-CREB signaling, the direct mechanism is unknown and remains to be elucidated. FXR thus appears to control the CREB signaling pathway in different tissues.

While the studies evaluating BA signaling or BA-FXR signaling in the CNS mainly focused on its potential role in neurodegenerative conditions (Keene et al., 2001, 2002; CastroCaldas et al., 2012; Lo et al., 2013; Vaz et al., 2015; Abdelkader et al., 2016; Elia et al., 2016; Moreira et al., 2017; Bell et al., 2018; McMillin et al., 2018; Rosa et al., 2018), very few papers 
investigated so far the role of FXR in brain control of energy homeostasis. A recent study reported that brain administration of the BA tauro-lithocholic acid (LCA) promotes fat oxidation and decreases fat mass associated with enhanced fatty acid uptake by BAT and browning of subcutaneous white adipose tissue (Eggink et al., 2018). Since LCA is a specific TGR5 agonist, without activity on FXR, these observations suggest opposite functions of the membrane BA receptor TGR5 and the nuclear receptor FXR. Similar contrasting roles of TGR5 and FXR have been observed previously with respect to the regulation of GLP1 secretion by L cells (Seyer et al., 2013; Trabelsi et al., 2015). In the same line, Castellanos-Jankiewicz's paper (CastellanosJankiewicz et al., 2021) show that the hypothalamic TGR5 activation by this BA mix induces an increased activity of the sympathetic nervous system (SNS) impacting energy expenditure without food intake or body weight changes, while our results demonstrate that hypothalamic activation of FXR induces a decreased activity of the SNS leading to a decreased energy expenditure. These results actually mirror those obtained in our study i.e., hypothalamic activation of FXR decreases activity of the SNS leading to a decreased energy expenditure. Data obtained in other organs, such as the intestine, have already revealed that FXR and TGR5 often exert antagonistic functions (Seyer et al., 2013; Trabelsi et al., 2015). It is likely that temporal differences in response to activators occur for TGR5 and FXR, the former upon activation transmitting its signal rapidly, whereas the former, acting as a transcription factor, allows a slower, more chronic response since this requires modulation of gene expression.

Thus, TGR5 and FXR likely also exert opposite actions on brain control of energy homeostasis to finely control, through the SNS, energy homeostasis and particularly energy expenditure. Overall, this implies that prevention of energy disorders would be achieved by stimulating TGR5 and/or inactivating hypothalamic FXR. Accordingly, Castellanos-Jankiewicz et al. show that the levels of TbMCA, one of the most abundant BA in plasma and in the hypothalamus and a known antagonist of FXR, strongly drop in DIO conditions.

Eggink et al. (2018) reported that ICV GW4064 (10 $\mu \mathrm{M}$ in final concentration in cerebrospinal fluid) did not modulate energy metabolism. The disparity of their results and ours may relate to the used dose, which in the present study was $3 \times$ greater than the one used by Eggink et al. Given the absence of precise data in the literature concerning the bioavailability and pharmacokinetics of GW4064 in the brain, it was crucial to perform preceding dose-response experiments in vivo to determine the efficient dose. Therefore, we first performed a doseresponse experiment with increasing doses of GW4064 by ICV. Similar as Eggink et al., at the dose of $10 \mu \mathrm{M}$ GW4064, we observed very limited effects of GW4064 on energy metabolism, which became pronounced when the GW4064 dose was increased to $30 \mu \mathrm{M}$, both with respect to significant changes of energy parameters as well as increased hypothalamic expression of FXR target genes and importantly, we could observe that our dose was selective to FXR activation since FXR deficiency completely abolished the effect of ICV GW4064 on UCP1 and TH protein expression in BAT as well as the induction of FXR and CREB target genes in the ARH.
Our study provides the first demonstration that brain FXR is an important central regulator of energy homeostasis. Our findings pave the way for further investigations on the regulatory role of FXR in the communication between the brain and peripheral organs and the control of energy metabolism. Moreover, studies on the role of tonic activation of hypothalamic FXR by its endogenous BA ligands under physiological or pathological (e.g., cholestatitc) conditions are warranted. Finally, determination of the contribution of brain FXR activation to the clinical actions of synthetic FXR ligands, several of which being currently in development for the treatment of metabolic diseases, such as non-alcoholic fatty liver disease, will be of interest.

\section{DATA AVAILABILITY STATEMENT}

The raw data supporting the conclusions of this article will be made available by the authors, without undue reservation.

\section{ETHICS STATEMENT}

The animal study was reviewed and approved by CEEA 75.

\section{AUTHOR CONTRIBUTIONS}

$\mathrm{BDc}, \mathrm{KB}$, and $\mathrm{BS}$ designed the research and wrote the manuscript. $\mathrm{BDc}, \mathrm{KB}, \mathrm{DD}, \mathrm{EN}, \mathrm{CB}$, and J-SA performed the experiments. EC performed the metabolic cages experiments. ED and SD participated in animal experiments. JC and BDp performed the GW4064 assay. CM performed and analyzed the sympathetic nerve activity recording. SL and AT analyzed the results. All authors contributed to the article and approved the submitted version.

\section{FUNDING}

This research was performed using funding provided by National Research Agency BABrain (ANR-17-CE14-0007), European Research Council Immunobile (694717) project and the Agence Nationale de la Recherche (ANR) grants "European Genomic Institute for Diabetes » E.G.I.D, ANR10-LABX-0046, a French State fund managed by ANR under the frame program Investissements d'Avenir I-SITE ULNE/ANR-16-IDEX-0004 ULNE.

\section{ACKNOWLEDGMENTS}

We thank M. Tardivel and A. Bongiovanni of the BioImaging Center (Lille University School of Medicine Research center) for their expertise and training us on the used devices. We thank C. Piveteau for the GW4064 assay. We thank V. Prevot and P. Giacobini for brain slices of NPY-gfp mice. We also thank the animal core facility (animal facilities of Univ. Lille-Inserm) 
of " Plateformes en Biologie Santé de Lille " and M. Besegher, J. Devassine, Y. Lepage, C. Meunier, C. Degraeve, and D. Taillieu for transgenic mice production and care.

\section{SUPPLEMENTARY MATERIAL}

The Supplementary Material for this article can be found online at: https://www.frontiersin.org/articles/10.3389/fnmol. 2021.808603/full\#supplementary-material

Supplementary Figure 1 | Effect of 6-days cerebral treatment with GW4064 on gene expression in BAT. mRNA expression of genes involved in glucose, lipid metabolism in BAT. The values are normalized to cyclophilin. Data are

\section{REFERENCES}

Abdelkader, N. F., Safar, M. M., and Salem, H. A. (2016). Ursodeoxycholic acid ameliorates apoptotic cascade in the rotenone model of Parkinson's disease: modulation of mitochondrial perturbations. Mol. Neurobiol. 53, 810-817. doi: 10.1007/s12035-014-9043-8

Abdelkarim, M., Caron, S., Duhem, C., Prawitt, J., Dumont, J., Lucas, A., et al. (2010). The Farnesoid X receptor regulates adipocyte differentiation and function by promoting peroxisome proliferator-activated receptor- and interfering with the Wnt/-catenin pathways. J. Biol. Chem. 285, 36759-36767. doi: 10.1074/jbc.M110.166231

Bantubungi, K., Prawitt, J., and Staels, B. (2012). Control of metabolism by nutrient-regulated nuclear receptors acting in the brain. J. Steroid Biochem. Mol. Biol. 130, 126-137. doi: 10.1016/j.jsbmb.2011.10.002

Bell, S. M., Barnes, K., Clemmens, H., Al-Rafiah, A. R., Al-ofi, E. A., Leech, V., et al. (2018). Ursodeoxycholic acid improves mitochondrial function and redistributes drp1 in fibroblasts from patients with either sporadic or familial Alzheimer's disease. J. Mol. Biol. 430, 3942-3953. doi: 10.1016/j.jmb.2018.08.019

Billinger, M., Seiler, C., Fleisch, M., Eberli, F. R., Meier, B., and Hess, O. M. (2001). Do beta-adrenergic blocking agents increase coronary flow reserve? J. Am. Coll. Cardiol. 38, 1866-1871. doi: 10.1016/S0735-1097(01)01664-3

Cannon, B. (2004). Brown adipose tissue: function and physiological significance. Physiol. Rev. 84, 277-359. doi: 10.1152/physrev.00015.2003

Cariou, B., Bouchaert, E., Abdelkarim, M., Dumont, J., Caron, S., Fruchart, J.-C., et al. (2007). FXR-deficiency confers increased susceptibility to torpor. FEBS Lett. 581, 5191-5198. doi: 10.1016/j.febslet.2007.09.064

Castellanos-Jankiewicz, A., Guzmán-Quevedo, O., Fénelon, V. S., Zizzari, P., Quarta, C., Bellocchio, L., et al. (2021). Hypothalamic bile acid-TGR5 signaling protects from obesity. Cell Metab. 33, 1483-1492.e10. doi: 10.1016/j.cmet.2021. 04.009

Castro-Caldas, M., Carvalho, A. N., Rodrigues, E., Henderson, C. J., Wolf, C. R., Rodrigues, C. M. P., et al. (2012). Tauroursodeoxycholic acid prevents MPTPinduced dopaminergic cell death in a mouse model of Parkinson's disease. Mol. Neurobiol. 46, 475-486. doi: 10.1007/s12035-012-8295-4

Chávez-Talavera, O., Tailleux, A., Lefebvre, P., and Staels, B. (2017). Bile acid control of metabolism and inflammation in obesity, Type 2 diabetes, dyslipidemia, and nonalcoholic fatty liver disease. Gastroenterology 152, 16791694.e3. doi: 10.1053/j.gastro.2017.01.055

Eggink, H. M., Tambyrajah, L. L., van den Berg, R., Mol, I. M., van den Heuvel, J. K., Koehorst, M., et al. (2018). Chronic infusion of taurolithocholate into the brain increases fat oxidation in mice. J. Endocrinol. 236, 85-97. doi: 10.1530/JOE-170503

Ek, L., and Åblad, B. (1971). Effects of three beta adrenergic receptor blockers on myocardial oxygen consumption in the dog. Eur. J. Pharmacol. 14, 19-28. doi: 10.1016/0014-2999(71)90118-X

Elia, A. E., Lalli, S., Monsurrò, M. R., Sagnelli, A., Taiello, A. C., Reggiori, B., et al. (2016). Tauroursodeoxycholic acid in the treatment of patients with amyotrophic lateral sclerosis. Eur. J. Neurol. 23, 45-52. doi: 10.1111/ene.12664 mean \pm SEM. ${ }^{*} P<0.05,{ }^{* *} P<0.01,{ }^{* * *} P<0.001$, Unpaired Student's $t$ test. Vehicle group is indicated as open bars, GW4064 group as black bars.

Supplementary Figure 2 | Effect of 6-days cerebral treatment with GW4064 on gene expression of BAT in an $8 \mathrm{~h}$-cold exposure paradigm. (A-E) Adra1a, Adra2a, Adrb3, Pgc1a, Dio2 mRNA expression in BAT of mice placed at room temperature $\left(23^{\circ} \mathrm{C}\right)$ or $4^{\circ} \mathrm{C}$. The values are normalized to cyclophilin or $18 \mathrm{~s}$. Data are mean \pm SEM. * ${ }^{*}$ VEH $23^{\circ} \mathrm{C}$ vs VEH $\left.4^{\circ} \mathrm{C}\right)$ \# (GW4064 $23^{\circ} \mathrm{C}$ vs GW4064 $\left.4^{\circ} \mathrm{C}\right)$ $P<0.05$, Two-Way ANOVA followed by Tukey post hoc. $23^{\circ} \mathrm{C}$ group is indicated as open bars, $4^{\circ} \mathrm{C}$ group as black bars.

Supplementary Figure 3 | Effect of 6-days cerebral treatment with GW4064 on FXR target gene expression in liver. (A,B) Shp and Bsep mRNA expression in liver by $q-P C R$. The values are normalized to cyclophilin. Data are mean \pm SEM.

${ }^{*} P<0.05,{ }^{* *} P<0.01,{ }^{* * *} P<0.001$, Unpaired Student's $t$ test. Vehicle group is indicated as open bars, GW4064 group as black bars.

Supplementary Table 1 | Primer for Sybr green and Taqman techniques.

Gofflot, F., Chartoire, N., Vasseur, L., Heikkinen, S., Dembele, D., Le Merrer, J., et al. (2007). Systematic gene expression mapping clusters nuclear receptors according to their function in the brain. Cell 131, 405-418. doi: 10.1016/j.cell. 2007.09.012

Hall, K. D., Heymsfield, S. B., Kemnitz, J. W., Klein, S., Schoeller, D. A., and Speakman, J. R. (2012). Energy balance and its components: implications for body weight regulation. Am. J. Clin. Nutr. 95, 989-994. doi: 10.3945/ajcn.112. 036350

Harms, M., and Seale, P. (2013). Brown and beige fat: development, function and therapeutic potential. Nat. Med. 19, 1252-1263. doi: 10.1038/nm.3361

Herzig, S., Long, F., Jhala, U. S., Hedrick, S., Quinn, R., Bauer, A., et al. (2001). CREB regulates hepatic gluconeogenesis through the coactivator PGC-1. Nature 413, 179-183. doi: 10.1038/35093131

Houssiere, A., Gujic, M., Deboeck, G., Ciarka, A., Naeije, R., and van de Borne, P. (2007). Increased metaboreflex activity is related to exercise intolerance in heart transplant patients. Am. J. Physiol. Heart Circ. Physiol. 293, H3699-H3706. doi: 10.1152/ajpheart.00694.2007

Huang, C., Wang, J., Hu, W., Wang, C., Lu, X., Tong, L., et al. (2016). Identification of functional farnesoid X receptors in brain neurons. FEBS Lett. 590, 3233-3242. doi: 10.1002/1873-3468.12373

Huang, F., Wang, T., Lan, Y., Yang, L., Pan, W., Zhu, Y., et al. (2015). Deletion of mouse FXR gene disturbs multiple neurotransmitter systems and alters neurobehavior. Front. Behav. Neurosci. 9:70. doi: 10.3389/fnbeh.2015. 00070

Keene, C. D., Rodrigues, C. M. P., Eich, T., Chhabra, M. S., Steer, C. J., and Low, W. C. (2002). Tauroursodeoxycholic acid, a bile acid, is neuroprotective in a transgenic animal model of Huntington's disease. Proc. Natl. Acad. Sci. U.S.A. 99, 10671-10676. doi: 10.1073/pnas.162362299

Keene, C. D., Rodrigues, C. M. P., Eich, T., Linehan-Stieers, C., Abt, A., Kren, B. T., et al. (2001). A bile acid protects against motor and cognitive deficits and reduces striatal degeneration in the 3-nitropropionic acid model of Huntington's disease. Exp. Neurol. 171, 351-360. doi: 10.1006/exnr.2001.7755

Kim, K.-S., Seeley, R. J., and Sandoval, D. A. (2018). Signalling from the periphery to the brain that regulates energy homeostasis. Nat. Rev. Neurosci. 19, 185-196. doi: $10.1038 /$ nrn. 2018.8

Lang, S. A., Maron, M. B., and Signs, S. A. (1989). Oxygen consumption after massive sympathetic nervous system discharge. Am. J. Physiol. Endocrinol. Metab. 256, E345-E351. doi: 10.1152/ajpendo.1989.256.3.E345

Lefebvre, P., Cariou, B., Lien, F., Kuipers, F., and Staels, B. (2009). Role of bile acids and bile acid receptors in metabolic regulation. Physiol. Rev. 89, 147-191. doi: 10.1152/physrev.00010.2008

Lo, A. C., Callaerts-Vegh, Z., Nunes, A. F., Rodrigues, C. M. P., and D'Hooge, R. (2013). Tauroursodeoxycholic acid (TUDCA) supplementation prevents cognitive impairment and amyloid deposition in APP/PS1 mice. Neurobiol. Dis. 50, 21-29. doi: 10.1016/j.nbd.2012.09.003

Maloney, P. R., Parks, D. J., Haffner, C. D., Fivush, A. M., Chandra, G., Plunket, K. D., et al. (2000). Identification of a chemical tool for the orphan nuclear receptor FXR. J. Med. Chem. 43, 2971-2974. doi: 10.1021/jm0002127 
McMillin, M., Grant, S., Frampton, G., Petrescu, A. D., Kain, J., Williams, E., et al. (2018). FXR-mediated cortical cholesterol accumulation contributes to the pathogenesis of type A hepatic encephalopathy. Cell. Mol. Gastroenterol. Hepatol. 6, 47-63. doi: 10.1016/j.jcmgh.2018.02.008

Moreira, S., Fonseca, I., Nunes, M. J., Rosa, A., Lemos, L., Rodrigues, E., et al. (2017). Nrf2 activation by tauroursodeoxycholic acid in experimental models of Parkinson's disease. Exp. Neurol. 295, 77-87. doi: 10.1016/j.expneurol.2017. 05.009

Narkiewicz, K., van de Borne, P., Montano, N., Hering, D., Kara, T., and Somers, V. K. (2006). Sympathetic neural outflow and chemoreflex sensitivity are related to spontaneous breathing rate in normal men. Hypertension 47, 51-55. doi: 10.1161/01.HYP.0000197613.47649.02

Pandey, S. C. (2003). Anxiety and alcohol abuse disorders: a common role for CREB and its target, the neuropeptide Y gene. Trends Pharmacol. Sci. 24, 456-460. doi: 10.1016/S0165-6147(03)00226-8

Piech-Dumas, K. M., and Tank, A. W. (1999). CREB mediates the cAMPresponsiveness of the tyrosine hydroxylase gene: use of an antisense RNA strategy to produce CREB-deficient PC12 cell lines. Mol. Brain Res. 70, 219-230. doi: 10.1016/S0169-328X(99)00149-7

Popescu, I. R., Helleboid-Chapman, A., Lucas, A., Vandewalle, B., Dumont, J., Bouchaert, E., et al. (2010). The nuclear receptor FXR is expressed in pancreatic beta-cells and protects human islets from lipotoxicity. FEBS Lett. 584, 28452851. doi: 10.1016/j.febslet.2010.04.068

Prawitt, J., Abdelkarim, M., Stroeve, J. H. M., Popescu, I., Duez, H., Velagapudi, V. R., et al. (2011). Farnesoid X receptor deficiency improves glucose homeostasis in mouse models of obesity. Diabetes Metab. Res. Rev. 60, 18611871. doi: $10.2337 / \mathrm{db} 11-0030$

Razzoli, M., Frontini, A., Gurney, A., Mondini, E., Cubuk, C., Katz, L. S., et al. (2016). Stress-induced activation of brown adipose tissue prevents obesity in conditions of low adaptive thermogenesis. Mol. Metab. 5, 19-33. doi: 10.1016/j. molmet.2015.10.005

Rosa, A. I., Duarte-Silva, S., Silva-Fernandes, A., Nunes, M. J., Carvalho, A. N., Rodrigues, E., et al. (2018). Tauroursodeoxycholic acid improves motor symptoms in a mouse model of Parkinson's disease. Mol. Neurobiol. 55, 9139 9155. doi: 10.1007/s12035-018-1062-4

Sánchez-Lasheras, C., Christine Könner, A., and Brüning, J. C. (2010). Integrative neurobiology of energy homeostasis-neurocircuits, signals and mediators. Front. Neuroendocrinol. 31, 4-15. doi: 10.1016/j.yfrne.2009.08.002

Seok, S., Fu, T., Choi, S.-E., Li, Y., Zhu, R., Kumar, S., et al. (2014). Transcriptional regulation of autophagy by an FXR-CREB axis. Nature 516, 108-111. doi: 10.1038 /nature 13949

Seyer, P., Vallois, D., Poitry-Yamate, C., Schütz, F., Metref, S., Tarussio, D., et al. (2013). Hepatic glucose sensing is required to preserve $\beta$ cell glucose competence. J. Clin. Invest. 123, 1662-1676. doi: 10.1172/JCI65538

Shimizu, I., Aprahamian, T., Kikuchi, R., Shimizu, A., Papanicolaou, K. N., MacLauchlan, S., et al. (2014). Vascular rarefaction mediates whitening of brown fat in obesity. J. Clin. Invest. 124, 2099-2112. doi: 10.1172/JCI71643

Sinal, C. J., Tohkin, M., Miyata, M., Ward, J. M., Lambert, G., and Gonzalez, F. J. (2000). Targeted disruption of the nuclear receptor FXR/BAR impairs bile acid and lipid homeostasis. Cell 102, 731-744. doi: 10.1016/S0092-8674(00)00062-3

Swanson, L. W., and Sawchenko, P. E. (1983). Hypothalamic integration: organization of the paraventricular and supraoptic nuclei. Annu. Rev. Neurosci. 6, 269-324. doi: 10.1146/annurev.ne.06.030183.001413

Trabelsi, M.-S., Daoudi, M., Prawitt, J., Ducastel, S., Touche, V., Sayin, S. I., et al. (2015). Farnesoid X receptor inhibits glucagon-like peptide-1 production by enteroendocrine L cells. Nat. Commun. 6:7629. doi: 10.1038/ncomms8629

Tully, D. C., Rucker, P. V., Chianelli, D., Williams, J., Vidal, A., Alper, P. B., et al. (2017). Discovery of Tropifexor (LJN452), a Highly Potent Non-bile
Acid FXR Agonist for the Treatment of Cholestatic Liver Diseases and Nonalcoholic Steatohepatitis (NASH). J. Med. Chem. 60, 9960-9973. doi: 10. 1021/acs.jmedchem.7b00907

Vaz, A. R., Cunha, C., Gomes, C., Schmucki, N., Barbosa, M., and Brites, D. (2015). Glycoursodeoxycholic acid reduces matrix metalloproteinase-9 and Caspase- 9 activation in a cellular model of superoxide dismutase-1 neurodegeneration. Mol. Neurobiol. 51, 864-877. doi: 10.1007/s12035-0148731-8

Watanabe, M., Horai, Y., Houten, S. M., Morimoto, K., Sugizaki, T., Arita, E., et al. (2011). Lowering bile acid pool size with a synthetic farnesoid X Receptor (FXR) agonist induces obesity and diabetes through reduced energy expenditure. J. Biol. Chem. 286, 26913-26920. doi: 10.1074/jbc.M111.248 203

Watanabe, M., Houten, S. M., Mataki, C., Christoffolete, M. A., Kim, B. W., Sato, H., et al. (2006). Bile acids induce energy expenditure by promoting intracellular thyroid hormone activation. Nature 439, 484-489. doi: 10.1038/nature04330

Waterson, M. J., and Horvath, T. L. (2015). Neuronal regulation of energy homeostasis: beyond the hypothalamus and feeding. Cell Metab. 22, 962-970. doi: 10.1016/j.cmet.2015.09.026

Witte, K. K. A. (2003). The effects of and blockade on ventilatory responses to exercise in chronic heart failure. Heart 89, 1169-1173. doi: 10.1136/heart.89. 10.1169

Witte, K. K. A., Notarius, C. F., Ivanov, J., and Floras, J. S. (2008). Muscle sympathetic nerve activity and ventilation during exercise in subjects with and without chronic heart failure. Can. J. Cardiol. 24, 275-278. doi: 10.1016/S0828282X(08)70176-4

Yang, L., and McKnight, G. S. (2015). Hypothalamic PKA regulates leptin sensitivity and adiposity. Nat. Commun. 6:8237. doi: 10.1038/ncomms 9237

Zhang, W., and Bi, S. (2015). Hypothalamic regulation of brown adipose tissue thermogenesis and energy homeostasis. Front. Endocrinol. 6:136. doi: 10.3389/ fendo.2015.00136

Zhang, Y., Ge, X., Heemstra, L. A., Chen, W.-D., Xu, J., Smith, J. L., et al. (2012). Loss of FXR protects against diet-induced obesity and accelerates liver carcinogenesis in ob/ob Mice. Mol. Endocrinol. 26, 272-280. doi: 10.1210/me. 2011-1157

Conflict of Interest: The authors declare that the research was conducted in the absence of any commercial or financial relationships that could be construed as a potential conflict of interest.

The reviewer DV declared a shared affiliation, though no other collaboration, with several of the authors, KB, BDc, DD, CB, SD, EN, ED, EC, JC, EV, J-SA, AT, BS, BDp, SL.

Publisher's Note: All claims expressed in this article are solely those of the authors and do not necessarily represent those of their affiliated organizations, or those of the publisher, the editors and the reviewers. Any product that may be evaluated in this article, or claim that may be made by its manufacturer, is not guaranteed or endorsed by the publisher.

Copyright (C) 2022 Deckmyn, Domenger, Blondel, Ducastel, Nicolas, Dorchies, Caron, Charton, Vallez, Deprez, Annicotte, Lestavel, Tailleux, Magnan, Staels and Bantubungi. This is an open-access article distributed under the terms of the Creative Commons Attribution License (CC BY). The use, distribution or reproduction in other forums is permitted, provided the original author(s) and the copyright owner(s) are credited and that the original publication in this journal is cited, in accordance with accepted academic practice. No use, distribution or reproduction is permitted which does not comply with these terms. 